\title{
Transient Three-Dimensional Geometrical/Thermal Modelling of Thermal Spray: Normal-Impinging Jet and Single Straight Deposits
}

\author{
F. Fanicchia ${ }^{\mathrm{a}}$, D. A. Axinte $\mathrm{a}^{\mathrm{a} *}$ \\ ${ }^{a}$ Rolls-Royce UTC in Manufacturing and On-Wing Technology, Faculty of Engineering, University of Nottingham, NG7 2RD, UK
}

\begin{abstract}
A new time-dependent approach to the geometrical and thermal modelling of the deposit footprint in thermal spray processes is proposed. Based upon a three-dimensional finite-difference numerical technique, the model is composed of two integrated sections: a geometrical analysis, accounting for deposit geometry analytical prediction, and a thermal analysis that computes the system temperature history. Primary process factors for the simulation, i.e. plume distribution parameters, jet heat transfer properties and temperature-dependent deposition efficiency are determined in a preliminary stage of model application. Through computation of the simulated impact surface temperature at each instant during the simulation, the deposition efficiency-mediated growth of the deposit is accurately predicted at arbitrary values of torch feed speed. The model is flexible as it only relies on an initial calibration stage performed at a specific set of process parameters to be able to predict deposition geometries at arbitrary conditions, thus avoiding the need of complex simulations and/or knowledge of single splats impact properties. Moreover, this modelling approach has the potential to be extended to several thermal spray processes at arbitrary values of process parameters (e.g. torch design, materials, etc.), opening the way for spray automation in difficult-to-spray geometries and/or repair applications. The proposed modelling framework has been validated on Combustion Flame Spray (CFS) deposition of CoNiCrAlY alloy on stainless steel substrates, yielding low errors $(<5 \%$ on average) in predicting the deposit footprint at various torch feed speeds.
\end{abstract}

\section{Introduction}

Thermal spray processes (e.g. plasma, high-velocity oxyfuel, flame spray, etc.) are commonly used to deposit a variety of materials over surfaces which provide them with additional specific properties 11. The material to be deposited is fed, in solid or solution form, into a highly energetic jet stream, hereafter named flame, which melts and propels it towards a target surface where it spreads and solidifies to generate a coating. Due to the high number of interacting factors affecting the deposition phenomenon, modelling the thermal spray process from first principles is inherently difficult. Additional difficulties are encountered because not all the material impacting the target surface contributes to coating generation but a deposition efficiency is instead observed, which is determined by the complex (thermal, geometrical and physico-chemical) nature of the interaction between impacting material and substrate [2, 3, 4].

An attempt to study interaction effects at dropletsubstrate impact is provided in the work of M. PasandidehFard et al., [5] where the impact deformation of the metal molten droplets is numerically and experimentally assessed

\footnotetext{
* Correspondingauthor

Email address: Dragos.Axinte@nottingham.ac.uk (D. A. Axinte)
}

against a variation in substrate preheat temperature. The observed variation in splat shape qualitatively demonstrates the dependency of the impact process on the surface temperature. Similar results are also presented in the work of Xue et al., 6]. Due to the dependency from several (interplaying) chemical-physical processes, to date, thermal spray deposition parameters for most industrial applications are empirically determined (trial-and-error approach). Consequently, automated deposition routines are reduced to simple substrate geometries (e.g. cylindrical) where the deposition hardware is thus maintained at a constant position/orientation in front of the rotating target. Thus, generally manual deposition is the routine adopted to deposit onto difficult-to-spray geometries (e.g. internal curved surfaces). Several developed thermal spray deposition models are focused on predicting the final deposit geometry starting from experimental observation on single particle impact. Models of this type are presented in the work of C.W Kang et al., and K. Remesh and al. for static (i.e. fixed torch position) deposit geometry prediction in plasma spray [7, 8]. Both approaches involve the combination of computational fluid dynamics to model the plasma flame and experimental observation on the shape of single splats to determine a relationship between the in-flight droplets parameters (e.g. speed, temperature) in the flame and the characteristics of the resulting overall deposit. Besides being comprehensive in simulating the 


\begin{tabular}{|c|c|c|c|}
\hline \multicolumn{4}{|c|}{ Nomenclature } \\
\hline & & $T^{E X P}$ & thermocouples-measured temperature \\
\hline$A$ & total constant spray distribution parameter & $T^{S I M}$ & simulated temperature \\
\hline$a$ & mass flux spray distribution parameter & $T_{\text {real }}$ & corrected thermocouple signal \\
\hline$b$ & mass flux spray distribution parameter & $T_{t c}$ & as-recorded thermocouple signal \\
\hline$C$ & specific heat & $t$ & time \\
\hline$D_{x 0}$ & torch position $\vec{G}$ initial x component & $V$ & torch feed speed \\
\hline$D_{y 0}$ & torch position $\vec{G}$ initial y component & $W_{0}$ & specimen initial weight \\
\hline$\Delta d$ & deposit/substrate mesh dimension, $\mathrm{x}$ and $\mathrm{y}$ axis & $W_{f}$ & specimen final weight \\
\hline$\delta d$ & deposit mesh dimension, $\mathrm{z}$ axis & $Z(r)$ & deposit profile cross-section \\
\hline$\dot{D}$ & deposition rate & \multicolumn{2}{|c|}{ Greek symbols } \\
\hline$d$ & assumed control volume thickness & $\alpha$ & emissivity \\
\hline$\dot{E}_{m e l t}$ & melting energy release term & $\epsilon$ & deposition efficiency \\
\hline$\hat{e}_{f}$ & surface unit vector connecting $\vec{P}$ and $\vec{G}$ & $\eta$ & droplet/impact surface interaction effects \\
\hline$\hat{e}_{n}$ & surface unit normal vector at $\vec{P}$ & $\theta$ & droplet impact angle \\
\hline$F$ & powder feed rate & $\rho$ & density \\
\hline$\vec{G}$ & torch position & $\sigma$ & Stefan-Boltzmann constant \\
\hline$\Delta H$ & increase in deposit height & $\tau$ & thermocouple response time \\
\hline$h$ & convection heat transfer & $\Psi$ & torch-to-substrate inclination \\
\hline$k$ & heat conductivity & \multicolumn{2}{|c|}{ Subscripts } \\
\hline$L$ & latent heat of melting & $A$ & atmospheric \\
\hline$l$ & specimen length & $D$ & deposit \\
\hline$\dot{m}$ & droplets spray mass flux & $E$ & edge \\
\hline$\vec{P}$ & surface impact point & int & interface \\
\hline$r$ & radial distance from torch axis & $J$ & flame \\
\hline$S O D$ & torch standoff distance & $Q$ & substrate \\
\hline$T$ & temperature & $S$ & surface \\
\hline
\end{tabular}

plasma spray process, models of this type present difficulties in accurately predicting temperature and chemistry of the complex flame flow through CFD, thus resulting in computationally expensive analysis. Because of this intrinsic complexity, significant difficulties arise on the applicability of these models for predicting complex flame paths (e.g. to spray complex 3D substrate geometries, for which CNC robots are the current industry standard). On the other hand, deposition models have also been developed where the impinging particles properties are simulated through a stochastic approach, as presented in the works of R. Ghafouri-Azar et al., and M. Xue et al. [9, 10]. These studies describe the in-flight particle properties as following normal (or log-normal) probability distributions. Coating formation is modelled following a set of a-priori defined droplet impact rules based on experimental observations on the final shape of impacted droplets. The main drawback of these approaches is their dependency on a specific set of deposition parameters, on which the defined empirical droplet impact rules are based. Moreover, due to the surface temperature-dependency of splat shape [11, the lack of heat transfer modelling and droplet-surface interaction effects in these models prevents the simulation of complex torch-surface relative motions from being sim- ulated. More recently, considerable attention has been given to the development of numerical methods, i.e. Lagrangian, Lagrangian-Eulerian and smooth particle hydrodynamics (SPH), mainly focused on the cold spray process [1, 13, 14, 15.

Although of different nature, but still linked to the impact of a stream of molten droplets, an interesting approach was reported by Mathur et al., on spray forming [16]. The study is based on a two-dimensional Gaussian formulation of the droplets deposition rate, this latter mediated by a deposition efficiency function which mainly depends on droplets-substrate thermal state and impact geometry. The validity of the analysis has been later supported by several works on spray casting [17, 18, all built over the framework of the aforementioned study. In practice however, due to fundamental discrepancies in the physics of the processes involved, these approaches would have limitations in the analysis of thermal spray processes. The absence of a high-energy density flame continuously impacting the deposition surface, together with the remarkably lower cooling rates measured in spray casting compared to thermal spray requires accurate modelling of the transient solidification kinetics (of both-in-flight and impacted material) in the former case. Conversely, the ex- 
tremely high cooling rates experienced in thermal spray processes allows the droplets to be assumed solid at impact, with the consequence that a thermal model of the process (essential to determine deposition efficiency) would not rely on solidification kinetics. Moreover, spray forming relies on simple deposition geometries, with minimal patterns of torch-substrate relative motion. The need to spray deposits by superposition of footprints (i.e. the full 3D geometry of the deposit) to enable coatings of accurate geometry on substrates of elaborated shape in thermal spray applications (e.g. curved internal surfaces or coating patches in repair applications) leads to complex geometricallydependent flame/spray stream/substrate thermodynamic interactions, not accounted for by the aforementioned works on spray forming.

To address these challenges, this paper presents a time-dependent modelling approach to predict the threedimensional footprint (i.e. deposit shape) and temperature for thermally-sprayed systems. In the model, a semiGaussian distribution is assumed for the droplet plume deposition rate, and a temperature-dependent deposition efficiency is also embedded. For that purpose, a threedimensional implicit finite-difference algorithm, based on two interplaying geometrical- and thermal-analysis sections, has been developed by selecting Combustion Flame Spray (CFS) as validation process. Model input parameters, including deposition rate, deposition efficiency function and flame thermal properties, are determined apriori through a novel iterative calibration-optimisation approach. The key advantage of the model is that it requires simple calibrations to capture the physics of the interaction between droplet plume and target surface for a specific set of process deposition parameters (e.g. flame flow, droplets feed rate, etc.). Subsequently, the deposit footprint can be predicted at arbitrary torch feed speed with little computational effort, thus allowing the method to be applicable for automation spraying on any system with continuous variation of torch feed speed. This flexibility is essential for dimensional control of deposit shape, as obtained from superposition of subsequent footprints from in-flight droplet spray distributions (hereafter named droplet plume), especially when difficult-to-spray geometries (e.g. curved internal surfaces) or small areas of delaminated coating (e.g. for repair applications) are required.

\section{Physical Background}

In order to capture the complexity of the interacting processes involved in thermal spray, a detailed description of their principle is here reported. A schematic of the CFS process is depicted in Fig. 1. Material in solid form, i.e powder, is injected within the torch, transported by a neutral carrier gas and then molten in-flight after entering the hot flame generated by flammable gas (e.g. acetylene) coupled with oxygen [19. Molten droplets flatten and overlap at impact with the substrate, generating

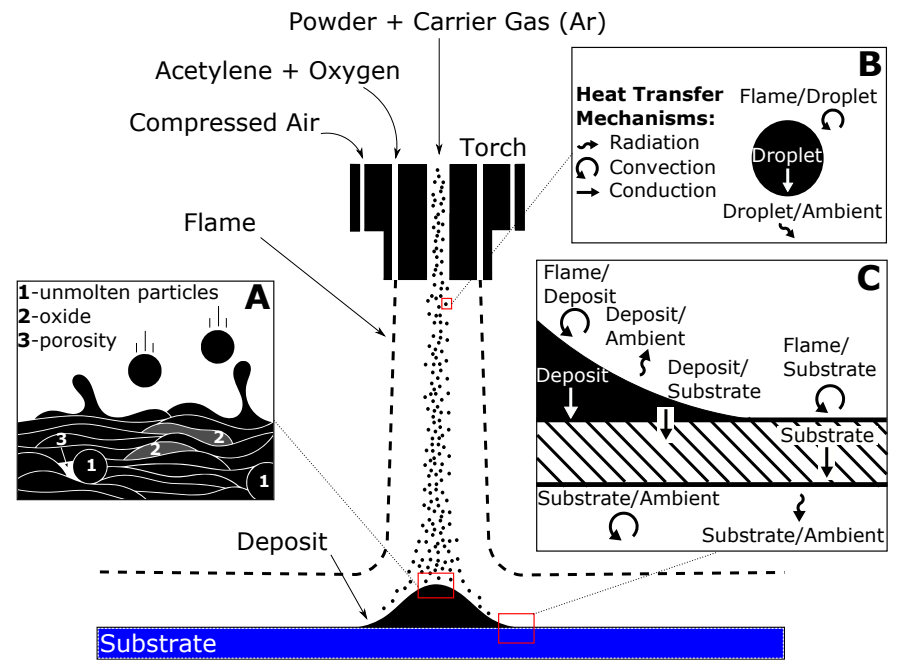

Figure 1: Schematic of the Combustion Flame Spray (CFS) process. The insets show (A) unmolten particles, oxide strings and porosity, typical deposit microstructural features; heat transfer mechanisms at in-flight droplet level (B) and deposit-substrate level (C).

a deposit of specific shape, i.e. the footprint. A sheath of compressed air at ambient temperature surrounds the flame to provide both additional kinetic energy to in-flight droplets and substrate cooling 11. The stochastic nature of the droplets plume in terms of particles temperatures, dimensions and velocity, involves complex impact flattening dynamics, with unmolten particles, oxides and porosity generated when multiple splats overlap (Fig. 1, inset A). Moreover, a given fraction of the impacting mass is scattered away from the target surface and does not contribute to deposit build-up. The ratio of mass retained over the total arriving at a given point can be referred to as deposition efficiency $(\epsilon)$. Modelling of this latter has found limited consideration in thermal spray literature and is generally confined to specific processes and deposition parameters [2, 3, 4, In general, deposition efficiency can be considered the result of two main contributions: (a) mechanical interaction between impacting droplets and the target surface (i.e. mechanical interlocking) and (b) target surface temperature (affecting diffusion-driven adhesion mechanisms).

Microscopically, the deposit footprint is dictated by both the properties of each impacting droplet (i.e. velocity, temperature, molten state, etc.) and the physicochemical interaction with the surface. Both properties are the result of the dynamic heat exchange history, i.e. time-dependence, during the process, both at a flame-todroplet level (Fig. 1, inset B), and flame-to-deposit (and substrate) level (Fig. 1, inset C); the latter also depending on the torch kinematic parameters (e.g. feed speed, inclination, standoff distance). At a droplet level, the primary heat-exchange mechanism is convective transfer with the flame, but radiation loss to the ambient and internal conduction within the droplet also have to be taken into account [20]. At the same time, mainly viscous drag, gravity and inertia forces define the droplets' velocity field [20]. 
Part of the heat stored within the droplets, together with radiative losses, is released to the underneath deposit (or substrate) at impact (Fig. 11 inset C). This heat can then be conducted through the deposit towards the substrate. At the same time, heat is exchanged, via convection, to the flame at the top surface and to the atmosphere at the bottom of the substrate.

The majority of the above-described heat-exchange processes involves interactions with the flame. Its properties, in terms of velocity and temperature fields, are rather complex. In fact, impinging gas jets used for thermal spray applications are typically turbulent in nature, with properties rapidly varying both along the standoff distance, caused by exchange of momentum with the surrounding air, and at impact with the substrate. Moreover, nozzles in thermal spray generally involve arrays of burners of varying layout with the consequence that multiple interacting flames have to be taken into account [21]. A comprehensive review of semi-analytical solutions of velocity field, heat and mass transfer for several array configurations of impinging gas jets is provided by Martin et al., 22. Effects of additional parameters as standoff distance [23, 24], and torch-to-substrate inclination [25] were also incorporated on a later stage. However, due to complexities in thermodynamics and kinetics of combustion chemistry, coupled with strong dependencies on the nozzle geometry, accurate modelling of the flame temperature field is generally hard to achieve in real applications [26]. For this reason, difficulties arise when attempting to model the temperature-dependent deposition efficiency function and, as a consequence, the deposit footprint.

The above complexities are addressed in this paper by capturing the majority of the above mentioned phenomena into a system calibration for a specific set of deposition parameters (that are usually kept constant spraying operations). In this way complex thermodynamic and geometrical factors are extracted, allowing a novel model for time-dependent deposit footprint to be simulated, opening the possibility to change continuously the shape of the deposit on arbitrary substrate shapes only by adjusting the torch kinematics.

\section{Model Development}

The analytical framework of the model, composed of interplaying geometrical-, thermal-analysis, along with a description of the methodology for characterising the necessary deposition parameters, is reported in the following paragraph. A numerical implementation of the model, is reported as a conclusive section.

\subsection{Geometrical analysis of the deposit}

The schematic of a succession of subsequent time steps during deposition, is depicted in Fig. 2. In the present work, the droplet plume flux distribution $\left(\dot{m},\left[\mathrm{~kg} / \mathrm{m}^{2} \mathrm{~s}\right]\right)$

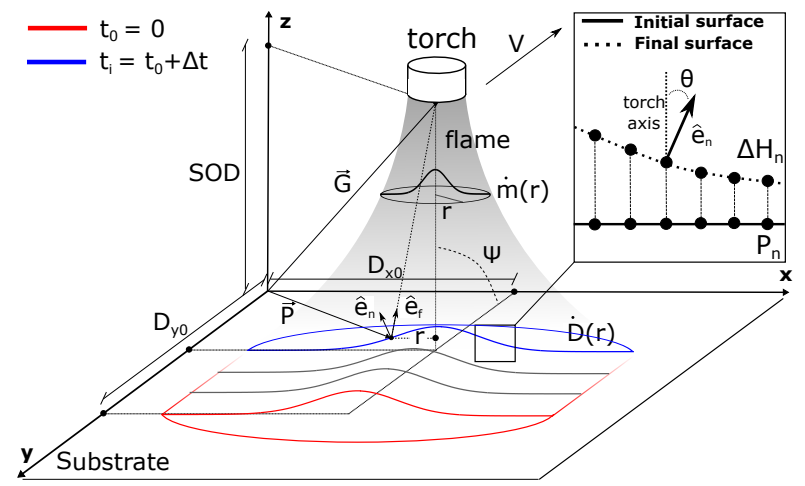

Figure 2: Schematic of factors involved in the geometrical analysis of the deposition process. The torch, with its axis oriented perpendicularly to the substrate surface and at fixed standoff distance (SOD), is considered moving at a feed speed $\mathrm{V}$ along the y-axis. The status of the process at an initial time $t_{0}=0$ (red) and at an arbitrary time $t_{1}=t_{0}+\Delta t$ (blue) is presented. The inset shows a detail of coating growth $\Delta H_{n}$ of $n$ points $P_{n}$ on the initially flat substrate surface, together with a definition of the droplet impact angle $\theta$ relative to the normal of the local point of the deposit.

is described by a Gaussian-shaped function of the form [16]:

$$
\dot{m}(r)=a \cdot e^{-b \cdot r^{2}}
$$

where a $\left(k g / m^{2} s\right)$ and $\mathrm{b}\left(1 / \mathrm{m}^{2}\right)$ are the plume distribution parameters, which depend on the set of deposition conditions used and are determined within the calibration stage in this work. However, these parameters vary with standoff distance (SOD) and torch-to-substrate inclination $(\psi)$ [16]. If these parameters are fixed during deposition, as it is commonly the case in spray operations and in thus in this work, the only dependency from the radial position from the torch axis $(r)$ is maintained as in the above equation. Once the droplet plume mass flux impacts the target surface, deposit grows at a rate defined by the deposition rate profile $(\dot{D},(\mathrm{~mm} / \mathrm{s}))$ :

$$
\dot{D}(r)=\epsilon\left(\eta, T_{s}\right) \cdot \dot{m}(r) \cdot \frac{1}{\rho_{D}},
$$

where $\rho_{D}\left(\mathrm{~kg} / \mathrm{m}^{3}\right)$ is the density of the growing deposit. Eq. 2 states that the droplet mass flux distribution is modified by a deposition efficiency function $\epsilon$ which depends on the mechanical- $(\eta)$ and chemical-interaction between impacting droplets and target surface that are driven by temperature-dependent diffusion phenomena. The term $\epsilon(\eta)$ represents the adhesion provided by mechanical interlocking between sprayed droplets and impact surface. It is thus determined by the particles flattening dynamics at impact with the target surface, influenced by droplets properties (temperature, velocity and impact angle $\theta$ ) as well as the micro-geometrical properties of the target surface (e.g. roughness). The impact surface temperature $T_{S}$, although also affecting the dynamics of droplet flattening, represents the major contribution to the thermallyactivated diffusion process for the droplet-target surface 
interface especially at the experimental conditions employed in this study (i.e. droplet-substrate materials of comparable chemistry and finely-polished substrate surface).

In this study, the torch is allowed to linearly move in the y-direction at feed speed $V$ (see Fig. 2). In Cartesian coordinates, after an elapsed amount of time $t_{i}=t_{0}+$ $\Delta t$ during deposition, the torch is located at an arbitrary position $\vec{G}=\left(\vec{G}_{x}, \vec{G}_{y}, \vec{G}_{z}\right)$ given by:

$$
\vec{G}=\left(D_{x 0}, \quad D_{y 0}+V \cdot \Delta t, \quad S O D\right)
$$

with $D_{x 0}$ and $D_{y 0}$ being the initial x and y components of the torch position respectively (i.e. at $t=t_{0}$, red profile in Fig. 2). At a time $t_{i}=t_{0}+\Delta t$ during deposition, droplets will hit a point $\vec{P}_{i}=\left(\vec{X}_{i}, \vec{Y}_{i}, \vec{Z}_{i}\right)$ on the impact surface (blue profile, Fig. 2). The initial surface is thus modified by the addition of new sprayed material as:

$$
\begin{aligned}
& X_{i}^{\prime}=X_{i}+\Delta H \cdot \hat{e}_{n} \cdot \hat{e}_{x} \\
& Y_{i}^{\prime}=Y_{i}+\Delta H \cdot \hat{e}_{n} \cdot \hat{e}_{y} \\
& Z_{i}^{\prime}=Z_{i}+\Delta H \cdot \hat{e}_{n} \cdot \hat{e}_{z}
\end{aligned}
$$

where, at each point under consideration $\hat{e}_{n}$ is the deposit/substrate surface unit normal vector, $\hat{e}_{x}, \hat{e}_{y}$ and $\hat{e}_{z}$ are the unit basis vectors and $\Delta H(r)[\mathrm{mm}]$ is the amount of material added, given by integrating the deposition rate, Eq. 2, in the time domain:

$$
\begin{aligned}
& \Delta H(r)=\int_{t}^{t+\Delta t} \dot{D}(r) \cdot \hat{e}_{n} \cdot \hat{e}_{f} d t \\
& =\int_{t}^{t+\Delta t} \frac{\epsilon\left(\eta, T_{s}\right)}{\rho_{D}} \cdot a e^{-b r^{2}} \cdot \hat{e}_{n} \cdot \hat{e}_{f} d t
\end{aligned}
$$

where $\hat{e}_{f}$ is the unit vector on the line connecting point $\vec{P}$ and torch position $\vec{G}$. This increment $\Delta H$ is schematically depicted in two-dimensions in the inset of Fig. 2.

In this work it is assumed that, at fixed values of standoff distance, torch inclination, flame gas flow properties and substrate preparation, the variables $\eta$ and $T_{S}$ on which the deposition efficiency term depend, represent independent contributions, e.g.: $\epsilon\left(\eta, T_{s}\right)=\epsilon(\eta) \cdot \epsilon\left(T_{S}\right)$. The mechanical interaction term $\epsilon(\eta)$, mainly depending on droplet properties at impact and target surface preparation, is thus independent from the deposition time at the fixed deposition conditions employed in this study and can be thus considered constant, i.e. $\epsilon(\eta)=$ const.. Moreover, as demonstrated by the low local impact angles obtained from the shallow deposits of this study, the dependency on the impact angle $\theta$, also embedded in this term, can be neglected [17. Finally, due to its dependency on the torch feed speed $V$ considered as the only parameter varied during this work, the surface temperature term $\epsilon\left(T_{S}\right)$ represents the only time-dependent contribution to the deposition efficiency. Eq. 5 would thus read:

$$
\Delta H(r)=A \int_{t}^{t+\Delta t} \epsilon\left(T_{S}\right) \cdot e^{-b r^{2}} \cdot \hat{e}_{n} \cdot \hat{e}_{f} d t
$$

where the factor $A=\epsilon(\eta) a / \rho_{D}$ has units of $\mathrm{m} / \mathrm{s}$. The above equation allows the spatially- and time-dependent deposit height increment $\Delta H(r)$ to be calculated, providing that plume distribution parameters $A$ and $b$ and surface temperature-dependent deposition efficiency term $\epsilon\left(T_{S}\right)$ are known.

In the present work, the plume distribution parameters are evaluated a-priori utilising a simple dedicated calibration stage, described in paragraph 3.3.2. This represents a reasonable approach since, as aforementioned, plume distribution parameters are constant at fixed values of standoff distance (SOD), torch-to-substrate inclination $(\Psi)$, deposit density $\left(\rho_{D}\right)$, and mechanical interaction term of deposition efficiency $(\epsilon(\eta))$ as assumed in this work. The surface temperature, affecting the deposition efficiency $\epsilon\left(T_{S}\right)$, is instead addressed through a thermal analysis of the deposition process, hereafter described.

\subsection{Thermal analysis of deposit and target surface, influ- encing deposition efficiency}

In order to predict the overall temperature of the substrate and deposit, a thermal analytical structure of the process is added to the geometrical analysis. Due to the time-dependent and geometrically complex nature of the deposit growth process, an analytical solution would be difficult to derive, and therefore a finite-differences method based on an energy balance of the heat diffusion equation is employed. In practice, the growing deposit/substrate system is subdivided into computational domains, each characterised by a different set of boundary conditions. Discrete elements are defined within each domain at different positions to cover the whole volume. A finite difference form of the heat diffusion equation is then obtained for each element of the different domains by applying the conservation of energy to a control volume about the nodal region [21. A cross-sectional schematic view (not in scale) of some the computational domains selected for a growing deposit and substrate is reported in Fig. 3 . It is worth noting that for the sake of simplicity only a few computational domains, both for deposit and substrate, are specified in the figure. A more detailed domain subdivision ( $\sim 80$ sub-domains), is in fact adopted in practical model application. The figure depicts new deposit material being sprayed at time $t_{0}+\Delta t$ (layer $\mathrm{D} 1$, bounded by red and blue dashed lines), added onto deposit previously sprayed at $t_{0}$ (layer $\mathrm{D} 2$, between blue and green dashed lines). A different colour coding is employed for the domains belonging to deposit material (D, black) and substrate (Q, blue). Flame (J, grey) and atmosphere (A, 


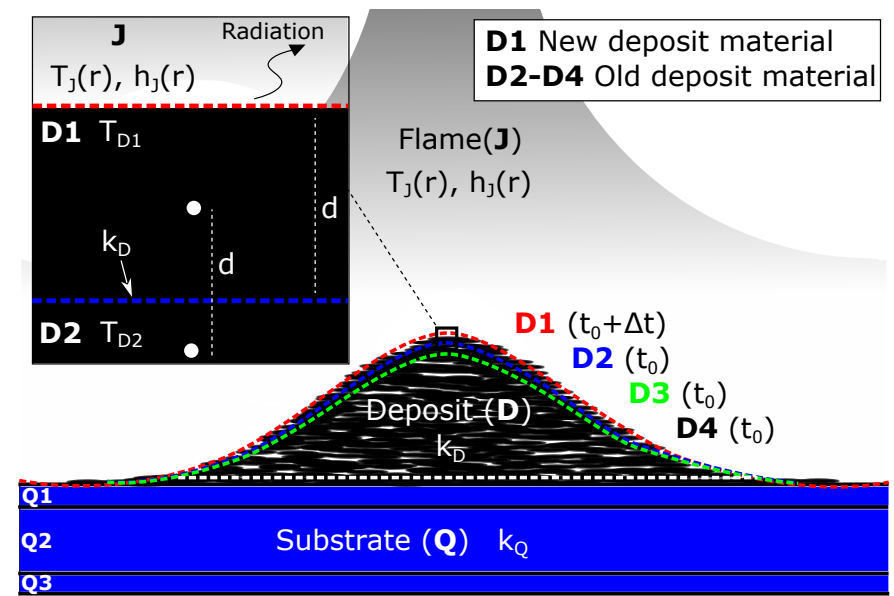

Atmosphere (A) $\mathrm{T}_{\mathrm{A}}, \mathrm{h}_{\mathrm{A}}$

Figure 3: Simplified 2D cross-sectional view of deposit (D) and substrate (Q) computational domains employed for thermal analysis. Boundary conditions are set by the flame (subscript $\mathrm{J}$, at the substrate top) and atmosphere (A, at the substrate bottom) in the form of gas surface temperature $T(r)$ and convective heat transfer coefficient $h(r)$. During this status of the process, a new layer of droplets (D1, bounded by the red dashed line) is added at the time step $t_{0}+\Delta t$ on top of the deposit already present at the previous time step $t_{0}$ (i.e. layers D2-D4). These latter are subdivided based on their boundary conditions: a thin layer immediately beneath the new deposit material (D2), material neither in contact with new deposit material nor substrate (D3) and material only in contact with the substrate (D4). Similarly, sub-domains are isolated within the substrate: elements in contact with deposit (Q1), elements internal to the substrate (Q2) and elements in contact with the atmosphere (Q3).

white), representing boundary conditions to the computational domains, are also specified. Within the deposit, several sub-domains are defined: new deposit material deposited at time step $t_{0}+\Delta t(\mathrm{D} 1)$, material sprayed at $t_{0}$ and in contact with new deposit material of layer D1 (D2), deposit material sprayed at $t_{0}$ internal to the deposit (D3) and material sprayed at $t_{0}$ only in contact with the substrate (D4). At substrate level the following domains are defined: a thin layer of material immediately underneath the deposit (Q1), internal material not in contact with either flame, deposit or atmosphere (Q2) and a thin layer of material in contact with the atmosphere (Q4). Irrespective of the spatial position $r$, incoming deposit (layer D1) is considered in molten state at a temperature $T_{D}^{0}$. Solidification is then taken into account by the release of latent energy from the incoming molten droplets to the deposit previously present on the target surface (i.e. from material of layer D1 to D2).

Deposit material, composed of overlapped droplets and thus containing pores, oxides and other defects, is substituted by discrete elements in the numerical simulation. The presence of these defects is not neglected but embedded within the conduction heat transfer coefficient $k_{D}$. The heat diffusion equation in the form of an energy balance is thus applied to the each elemental node based on the specific set of boundary conditions around it. The situation of an element belonging to layer D1, simplified assuming 1D heat transfer only in the vertical direction (no transfer to confining side elements) is depicted in the inset of Fig. 3. As depicted in the inset, in this work the inter-elements spacing in the vertical direction is assumed corresponding to the element thickness $d$. This latter value assumes a different value for substrate, deposit elements and at the interface between the two (Par. 3.4). The element represents new deposit being just added and thus exchanges heat through convection with the flame and by conduction with the underlying element of the deposit already present on the target surface (D2). Additional heat is dissipated to the atmosphere by means of radiation. The heat diffusion equation in this case would thus read:

$$
\begin{aligned}
\text { D1) } & h_{J}(r)\left(T_{D 1}-T_{J}(r)\right)-\frac{k_{D}\left(T_{D 1}-T_{D 2}\right)}{d} \\
& -\alpha_{D} \sigma\left(T_{D 1}^{4}-T_{A}^{4}\right)=\rho_{D} C_{D} d \frac{d T_{D 1}}{d t}
\end{aligned}
$$

where the three terms in the left-side represent flame convection, conduction with the layer D2 and the radiative loss while the right side of the equation is the rate of thermal energy variation within the element thickness (or volume in three-dimensions). Parameters $\alpha_{D}(-)$, $\rho_{D}\left(\mathrm{~kg} / \mathrm{m}^{3}\right)$ and $C_{D}(\mathrm{~J} / \mathrm{kgK})$ are coating emissivity, density and specific heat respectively, while $\sigma$ is the Boltzmann constant. Terms $T_{J}(r)(K)$ and $h_{J}(r)\left(W / m^{2} K\right)$ are flame temperature and convective heat transfer coefficient at the deposit surface and depend spatially on the radial position from the flame axis (Fig. 2). By applying the same energy balance methodology to the other computational domains thus gives:

(D2)

$$
\begin{aligned}
& \frac{k_{D}\left(T_{D 2}-T_{D 1}\right)}{d}-\frac{k_{D}\left(T_{D 2}-T_{D 3}\right)}{d} \\
& +\dot{E}_{\text {melt }}=\rho_{D} C_{D} d \frac{d T_{D 2}}{d t}
\end{aligned}
$$

(D3)

$$
\begin{aligned}
& \frac{k_{D}\left(T_{D 3}-T_{D 2}\right)}{d}-\frac{k_{D}\left(T_{D 3}-T_{D 4}\right)}{d} \\
& =\rho_{D} C_{D} d \frac{d T_{D 3}}{d t}
\end{aligned}
$$

(D4)

$$
\begin{aligned}
& \frac{k_{D}\left(T_{D 4}-T_{D 3}\right)}{d}-\frac{k_{i n t}\left(T_{D 2}-T_{Q 1}\right)}{d} \\
& =\rho_{D} C_{D} d \frac{d T_{D 4}}{d t}
\end{aligned}
$$

(Q1)

$$
\begin{aligned}
& \frac{k_{i n t}\left(T_{Q 1}-T_{D 4}\right)}{d}-\frac{k_{Q}\left(T_{Q 1}-T_{Q 2}\right)}{d} \\
& =\rho_{Q} C_{Q} d \frac{d T_{Q 1}}{d t}
\end{aligned}
$$

(Q2)

$$
\begin{aligned}
& \frac{k_{Q}\left(T_{Q 2}-T_{Q 1}\right)}{d}-\frac{k_{Q}\left(T_{Q 2}-T_{Q 3}\right)}{d} \\
& =\rho_{Q} C_{Q} d \frac{d T_{Q 2}}{d t}
\end{aligned}
$$




$$
\text { (Q3) } \begin{aligned}
& \frac{k_{Q}\left(T_{Q 3}-T_{Q 2}\right)}{d}-h_{A}\left(T_{Q 3}-T_{A}\right) \\
& -\alpha_{Q} \sigma\left(T_{Q 3}^{4}-T_{A}^{4}\right)=\rho_{Q} C_{Q} d \frac{d T_{Q 3}}{d t}
\end{aligned}
$$

The heat exchange at the deposit-substrate interface (layers D4-Q1) is accounted for through the interface conductivity $k_{\text {int }}(W / m K)$ (Eqs. 10 11). Assuming conduction as dominant heat transfer mechanism at the depositsubstrate interface, this has been here calculated by using a simple mixing rule between the values of deposit and substrate as $k_{i n t}=2 k_{D} k_{Q} /\left(k_{D}+k_{Q}\right)$. It is worth noting that both a radiative dissipation and convective heat transfer terms to the atmosphere (assumed of constant temperature $T_{A}$ and convective heat transfer coefficient $h_{A}$ ) are active in layer Q3 (Eq. 13). Moreover, exchange of melting energy from layer D1 to D3 is included through the positive energy term $E_{\text {melt }}=L_{D} \rho_{D} d$ (Eq. 8), with $L_{D}(J / k g K)$ latent heat of melting.

The addition of the above described thermal framework to the geometrical analysis previously portrayed allows the target surface temperature $T_{S}$ in Eq. 6. on which the deposition efficiency term $\epsilon$ depends, to be determined. This thermal analysis, however, relies on the knowledge of flame temperature immediately above the target surface $\left(T_{J}(r)\right)$ and convective heat transfer coefficient $\left(h_{J}(r)\right)$, both showing a behaviour that varies with radial distance from the torch axis (r) according to torch design, flame turbulence, standoff distance, target surface roughness, etc. Moreover, in order to make use of Eq. 6, plume distribution parameters $A$ and $b$, and surface temperaturedependent deposition efficiency distribution $\left(\epsilon\left(T_{S}\right)\right.$ must be known for the specific set of deposition parameters employed. This is the objective of the model parameters evaluation stage, hereafter described.

\subsection{Model parameters evaluation}

Flame convective heat transfer coefficient $h_{J}(r)$ and target surface temperature $T_{J}(r)$ in Eq. 7. required for thermal-analysis application, together with plume distribution parameters $A$ and $b$ and the temperaturedependent deposition efficiency function $\epsilon\left(T_{S}\right)$ in Eq. 6 are here determined. At this scope, the following evaluation stages are employed: flame characterisation (for $T_{J}(r)$ and $h_{J}(r)$ determination, Par. 3.3.1), calibration (for $A$ and $b$ calculation, Par. 3.3.2 and deposition efficiency evaluation (for $\epsilon\left(T_{S}\right)$ analysis, Par. 3.3.3). The spray process is here simplified by considering flame and droplet plume as separate domains or, in other words, the flame thermodynamic properties (i.e. temperature and flow) are not considered to be affected by the presence of in-flight particles during deposition. Thus the flame properties $\left(T_{J}(r)\right.$ and $\left.h_{J}(r)\right)$ could be characterised without computationally-expensive CFD models including in-flight flame-powder interactions, but rather semi- empirically through direct analysis of substrate temperature variation from flame impingement. Similarly, the shape of the droplet deposition rate, defined by the plume distribution parameters $A$ and $b$, can be directly obtained from measurement of the droplet plume footprint over the deposition surface, without the need of complex models based on in-flight droplet size distribution and thermodynamic history, which is commonly employed to model similar spray processes.

A detailed description of the three above described evaluation stages is hereafter reported.

\subsubsection{Flame characterisation $\left(h_{J}(r)\right.$ and $T_{J}(r)$ boundary conditions)}

A novel semi-empirical method is employed in this work to determine the flame convective heat transfer coefficient $h_{J}(r)$ and temperature at surface $T_{J}(r)$ parameters, necessary to implement the thermal analysis (Eq. 7). The assumed separation of flame and droplet plume domains allows the flame parameters to be determined from simple experiments involving only the flame, with no powder injected. In particular, in this approach, the torch is ignited and kept static in front of a substrate for a defined period, with its axis aligned with the substrate centre. The substrate surface temperature is then measured by means of an array of thermocouples, positioned at several radial positions from the centre. A detailed description of the experimental setup is found in Par. 4.1.1. Results from the experiments are then compared, within an optimisation algorithm, to the outcomes of numerical simulations replicating the experiments, utilising the flame properties as variable inputs.

In practice, the substrate surface temperature from the experiments $\left(T_{S}^{E X P}(r, t)\right)$ is measured at specific radial locations from the flame axis and compared to the numerical simulation output of the same quantity at the same radial locations $\left(T_{S}^{S I M}(r, t)\right.$, Fig. 4), thus in a point-wise approach. It is worth specifying that, $T_{J}(r)$ represents the temperature of the flame at the substrate surface while $T_{S}^{E X P}(r, t)$ is the actual temperature of the substrate surface. A new value of $T_{S}^{S I M}(r, t)$ is then obtained at each iteration step within an optimisation loop by varying the set of input flame convective heat transfer coefficient $\left(h_{J}^{i}(r)\right)$ and temperature at surface $\left(T_{J}^{i}(r)\right)$ until a set convergence criteria $T_{S}^{E X P}(r, t)-T_{S}^{S I M}(r, t)<\delta$ is met.

Theoretically, a static flame impinging onto a substrate would be represented similarly to Fig. 3 while removing the domains corresponding to the deposit (D1-D4). The application of the energy balance in this case would result in differential equations similar to Eqs. 11.13. where the equation corresponding to domain Q1 would now contain an additional convective heat transfer term $\left(h_{J}(r)\left(T_{Q 1}-\right.\right.$ $\left.T_{J}(r)\right)$ ) while neglecting the interface heat transfer term 


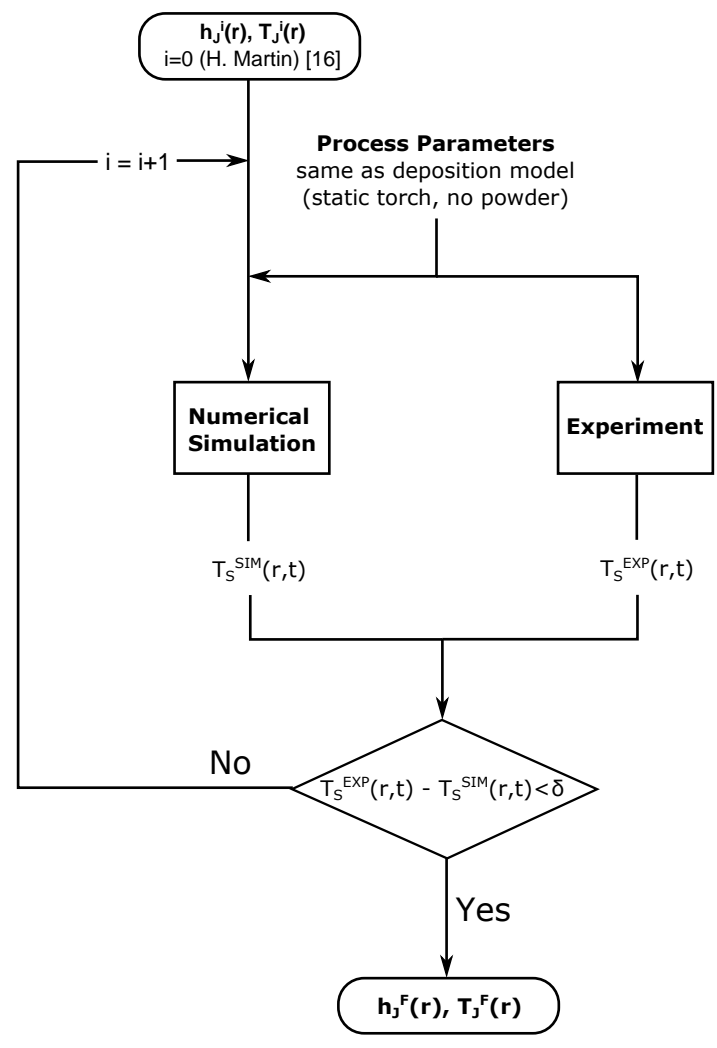

Figure 4: Schematic of the optimisation algorithm implemented for flame characterisation. The final flame convective heat transfer coefficient $\left(h_{J}^{F}(r)\right)$ and temperature at surface $\left(T_{J}^{F}(r)\right)$ are determined by minimising the difference between simulated $\left(T_{S}^{S I M}(r, t)\right)$ and experimental $\left(T_{S}^{E X P}(r, t)\right)$ time dependent substrate temperature profiles.

$\left(\frac{k_{i n t}\left(T_{Q 1}-T_{D 4}\right)}{d}\right):$

$$
\begin{array}{cl}
\left(\mathbf{Q 1} 1^{\text {new }}\right) & h_{J}(r)\left(T_{Q 1}-T_{J}(r)\right)-\frac{k_{Q}\left(T_{Q 1}-T_{Q 2}\right)}{d} \\
& =\rho_{Q} C_{Q} d \frac{d T_{Q 1}}{d t}
\end{array}
$$

An unconstrained non-linear optimisation method, based on the Simplex search algorithm 27] has been used with $h_{J}^{i}(r)$ and $T_{J}^{i}(r)$ as variables and the difference between experimental and numerical substrate surface temperatures $T_{S}^{E X P}(r, t)-T_{S}^{S I M}(r, t)$ as minimisation function. As starting point for the simulation, the convection heat transfer coefficient $h_{J}^{0}(r)$ was determined based on the work of Martin H. on impinging gas jets [22]. For the same coefficient, the flame properties have been adapted from 28. with a constant temperature $T_{J}^{0}(r)=\left(T_{\text {adiab }}+T_{A}\right) / 2$ imposed ( $T_{\text {adiab }}=3410 \mathrm{~K}$ is the flame adiabatic temperature of an oxyacetylene flame [29]). At each iteration $i+1$, the variables values are modified until the convergence criteria has been met, thus providing the final parameters $h_{J}^{F}(r)$ and $T_{J}^{F}(r)$.

\subsubsection{Calibration (plume distribution parameters $A$ and} b)

In this stage, the plume distribution parameters $A$ and $b$ in Eq. 6] are determined. The procedure involves the experimental generation of a single deposit at the same set of process parameters employed for the model numerical predictions, except maintaining an arbitrarily high torch feed speed to ensure shallow deposits, as required to determine the deposition rate profile $(\dot{D}(r)$, as hereafter detailed). This methodology could be adopted since, as experimentally and numerically observed in this work for a deposit at specific feed speed $\left(V^{i}\right)$, the temperature profile of the target surface $\left(T_{S}^{i}\right)$ in the area of deposit growth at each instant of time during torch movement can be assumed roughly constant (refer to the discussion connected to Fig. 5p. With this assumption, a unique temperature $T_{S}^{i}$ can be associated to the specific torch feed speed $V^{i}$. This allows a constant value of the deposition efficiency factor $\epsilon\left(T_{S}\right)$ in Eq. 6 to be employed. This latter is then directly calculated by measuring the substrate weight before $\left(W_{0}\right)$ and after $\left(W_{f}\right)$ deposition, through the relationship:

$$
\epsilon\left(T_{S}^{i}\right)=\epsilon\left(V^{i}\right)=\frac{W_{f} V^{i}}{W_{0} V^{i}+F l}
$$

where $F(\mathrm{~g} / \mathrm{min})$ is the powder feed rate and $l(\mathrm{~m})$ is the substrate width. The obtained deposit is thus scanned and its two-dimensional cross-section extracted. From the obtained profile, the experimental deposition rate profile $\dot{D}(r)$ (Eq. 2 is then extracted and least-square fitted to Eq. 6 to obtain the plume distribution parameters $A$ and $b$. In order to obtain the deposition rate profile from an experimental deposit footprint cross-section, a modified version of the work of Axinte et al., on the water-jet erosion model [30] is developed in this study. In the work, the erosion rate profile $\dot{E}(r)$ is determined by inverting the expression for water-jet-generated erosion footprint $Z(r)$. By applying a similar methodology to the deposition process of this study, replacing erosion rate with deposition rate $\dot{D}(r)$ thus gives:

$$
\dot{D}(r)=-\frac{1}{\pi}\left\{\int_{r}^{1} \frac{(Z(s)-Z(r))}{\left(s^{2}-r^{2}\right)^{3 / 2}} d s-\frac{Z(r)}{\left(1-r^{2}\right)^{1 / 2}}\right\},
$$

where $Z(r)$ in this case represents the deposit profile crosssection. It is worth noting that the above equation is only valid in case of shallow deposit profiles $Z(r)$; therefore its accuracy is improved by increasing the torch feed speed $V$.

From knowledge of the weighted surface-temperature dependent deposition efficiency $\epsilon\left(T_{S}^{i}\right)$, the obtained deposition rate profile $\dot{D}(r)$ is fitted to its semi-Gaussian expression (Eq. 2 to obtain the required plume distribution parameters $A=\epsilon(\eta) a / \rho_{D}$ and $b$.

\subsubsection{Deposition Efficiency evaluation $\left(\epsilon\left(T_{S}\right)\right)$}

In this stage, the continuous function $\epsilon\left(T_{S}\right)$ in Eq. 6 is determined. The methodology consists in producing two 
single straight deposits sprayed at the low and high boundary of torch feed speeds (compared to the values employed in application), $V^{1}$ and $V^{f}$, with subsequent deposition efficiency $\epsilon\left(V^{i}\right)$ determined according to weight measurements (Eq. 15). For each of the two deposits of torch feed speed $V^{i}$ corresponding constant target surface temperature $T_{S}^{i}$ are measured from numerical simulations of the same depositions, with plume distribution parameters $A$ and $b$ previously determined within the calibration stage. This constant target surface temperature could be determined from the observation that at each time step during deposition the surface temperature assumes a roughly equivalent distribution within the deposition region around the torch axis. The width of this deposition region, determined from knowledge of the previously calculated deposition rate $(\dot{D}(r)$, Eq. 16, is depicted in Fig. 5 for the first instant of deposition (i.e. $\Delta t_{1}=0.12 \mathrm{~s}$ ) of a deposit sprayed at $V=10 \mathrm{~mm} / \mathrm{s}$ and time increments $\Delta t=0.12 \mathrm{~s}$. In the figure, the line over which the profiles

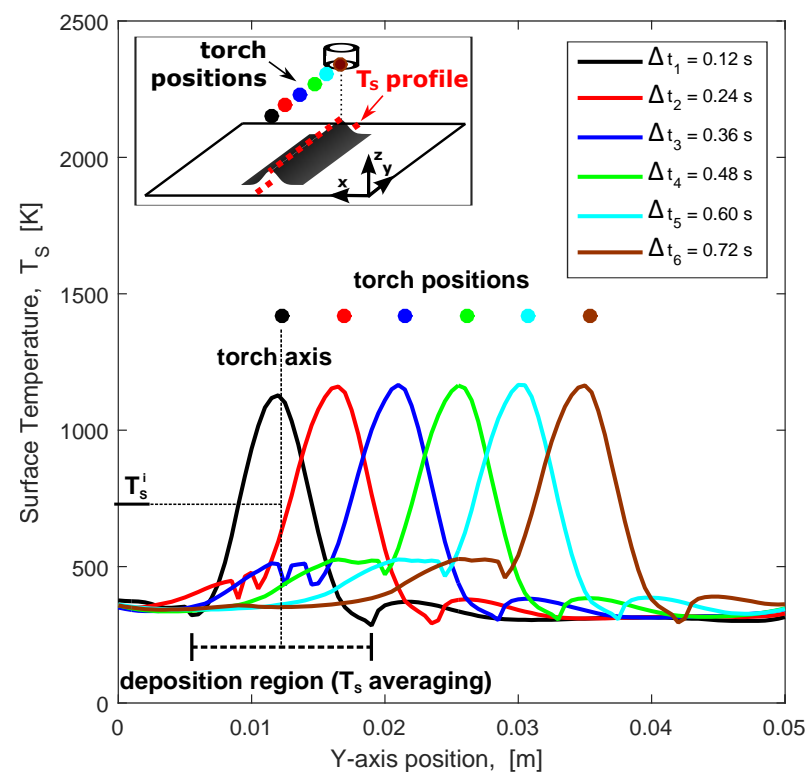

Figure 5: Simulated surface temperature profiles $\left(T_{S}\right.$, red dotted line in inset) and torch positions, colour-coded for subsequent time increments $\Delta t_{1}=0.12 \mathrm{~s}$, demonstrating comparable magnitudes within the area of deposition (i.e. deposition region, around the torch axis as determined from the deposition rate $\dot{D}(r)$ in Eq. 16 . The constant surface temperature profile $T_{S}^{i}$ corresponding to the torch feed speed $V^{i}$ of deposition is determined as a mean between the average value at each time step.

have been probed is depicted as a red dashed line (inset) and the torch position at each time step is denoted by a color-coded dot over each temperature profile. It is worth noting that comparable temperature profiles are recorded at each instant of time, confirming the aforementioned assumption.

From the weighted deposition efficiencies $\epsilon\left(V^{i}\right)$ and the constant surface temperatures measured, a correlation to two discrete $\epsilon\left(T_{S}^{i}\right)$ values is then obtained. The two deposition efficiencies are then plotted and linearly fitted to an equation of the form $\epsilon\left(T_{S}\right)=a \cdot T_{S}+b$ to obtain a continuous function of target surface temperature.

With continuous deposition efficiency $\left(\epsilon\left(T_{S}\right)\right)$ and plume distribution parameters $(A, b)$ so determined, Eq. 6 can be then employed to predict deposit geometry at arbitrary values of torch feed speed. The numerical implementation of the model, combining geometrical- and thermal analysis, is hereafter described.

\subsection{Numerical Implementation}

A schematic summary of the model framework is depicted in Fig. 6. The factors needed for the implementation, i.e. plume distribution parameters ( $A$ and $b)$, deposition efficiency function $\left(\epsilon\left(T_{S}\right)\right)$ and flame boundary conditions $\left(h_{J}(r)\right.$ and $\left.T_{J}(r)\right)$ are obtained, a priori, within the model parameters evaluation phase. By employing the same set of process parameters (except a variable feed speed), these factors are then fed into the numerical implementation of geometrical- and thermal-analysis, mutually cooperating to determine deposit height $(\Delta H)$ and target surface temperature $\left(T_{S}\right)$ represents the core of the model, based on Eq. 6.

At this scope, an implicit finite-difference algorithm was implemented in Matlab ${ }^{T M}$. The computational domains, substrate and growing deposit, have been discretised using hexahedral elements of dimensions $\Delta d \times \Delta d \times \Delta d=$ $0.5 \times 0.5 \times 0.5 \mathrm{~mm}^{3}$ and $\Delta d \times \Delta d \times \delta d=0.5 \times 0.5 \times 0.001 \mathrm{~mm}^{3}$ respectively (Fig. 7). In order to replicate the experimental setup, $50 \times 50 \times 6 \mathrm{~mm}^{3}$ has been selected as substrate dimensions and deposits have been generated by moving the torch $30 \mathrm{~mm}$ over the substrate $(x=$ const. $=0.025$, from $y=0.01$ to $y=0.04 \mathrm{~m}$ ). A refined mesh has been employed at the interface within both domains for accuracy improvement purposes, while an iteration time step $\Delta t=0.001 s$ has been selected.

Elements within the deposit mesh (or ghost grid) are only activated during deposition, based on the analytical profile predicted by the geometrical model (determined from Eq. 6). At each iteration time step, based on the thermal boundaries of each of the $\mathrm{N}$ (deposit and substrate) elements currently active, a set of $\mathrm{N}$ finitedifference equations is then assembled (based on Eqs. 7 . 13). At each time step $t$ during the simulation, the system of differential equations is thus solved to obtain the nodal temperatures for the subsequent iteration time step $t+\Delta t$.

A schematic of the steps involved in the model numerical implementation is depicted in Fig. 8.

- STEP 1. At the initial stage of simulation $(t=$ $\left.t_{0}\right)$ The computational domains (substrate and deposit) are discretized and prepared for the coating growth prediction step. A grid of nodes at positions $X_{0}(i), Y_{0}(i), Z_{0}(i)$ is thus created at the substrate surface, representing the plane where the deposit growth will take place. Above the substrate, a "ghost" mesh is generated, matching in underlying substrate surface elements in $\mathrm{x}-\mathrm{y}$ dimensions. At each time iteration the 


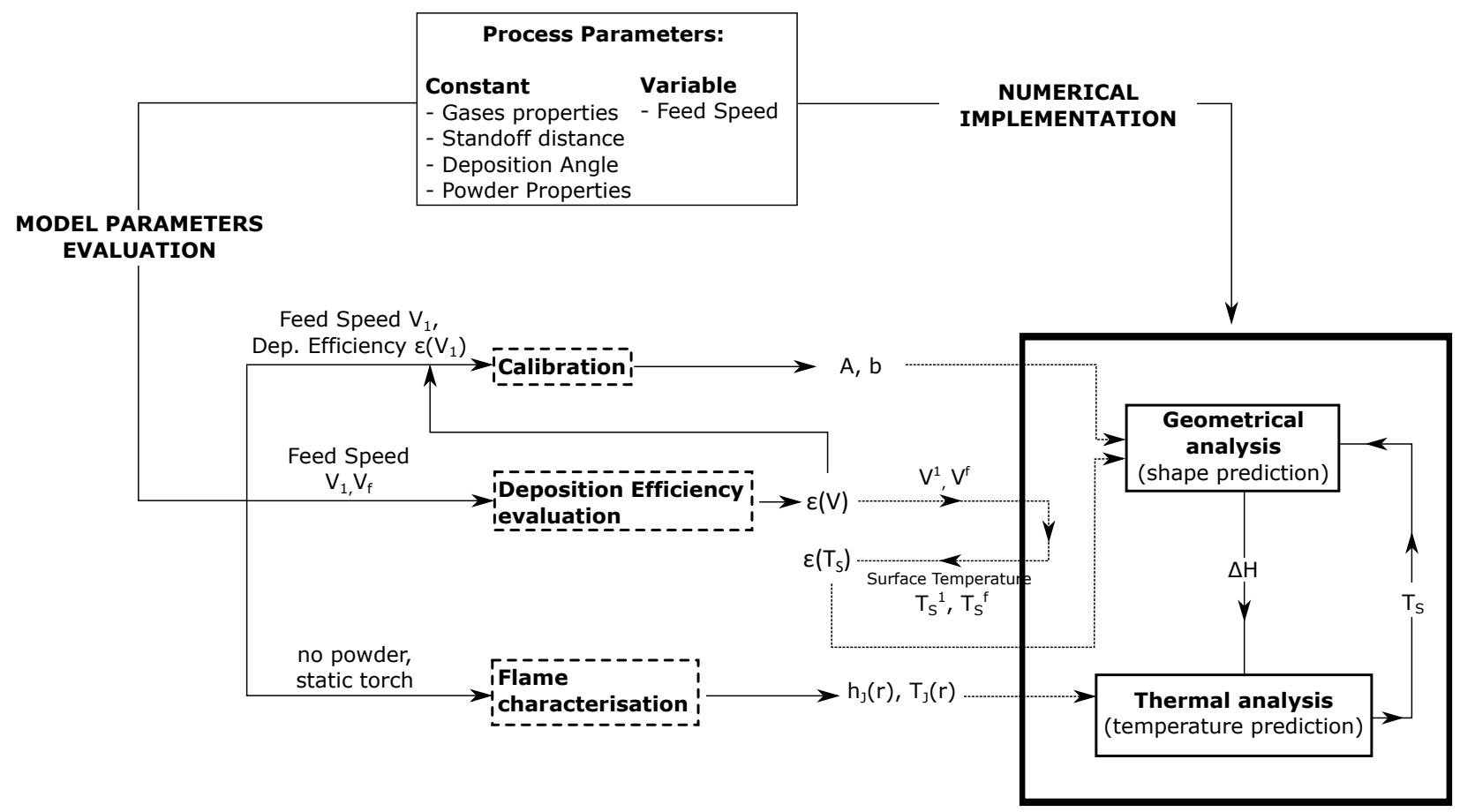

Figure 6: Schematic diagram of the modelling framework. Deposit footprint predictions at arbitrary torch feed speeds are performed within the Numerical Implementation of geometrical- (for deposit height $\Delta H$ ) and thermal-analysis (for surface temperature $T_{S}$ ). Factors necessary for the numerical implementation are determined, at the same set of constant process parameters, within the Model Parameters Evaluation stage: plume distribution parameters $\left(A\right.$ and $b$, Calibration), deposition efficiency function $\left(\epsilon\left(T_{S}\right)\right.$, Deposition Efficiency Evaluation) and flame properties $\left(h_{J}(r)\right.$ and $T_{J}(r)$, Flame Characterisation).

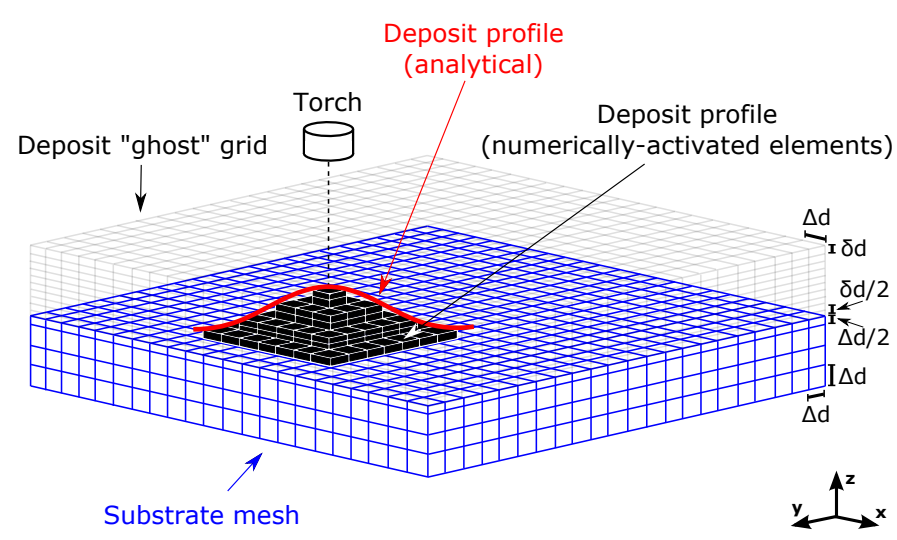

Figure 7: Computational domains used for model numerical implementation. Only those elements of the deposit ghost grid (grey) underneath the analytically-determined profile (red) are activated (black) at each time increment. Hexahedral elements of $0.5 \times 0.5 \times$ $0.5 \mathrm{~mm}^{3}$ (substrate, blue) and $0.5 \times 0.5 \times 0.001 \mathrm{~mm}^{3}$ (deposit) have been employed for a total $50 \times 50 \times 6 \mathrm{~mm}^{3}$ substrate size.

deposit mesh works in a way that only its elements whose combination fills the shape of the deposit predicted by the geometrical model are active. At the beginning of the simulation, there are therefore no active elements within the deposit mesh. A uniform initial substrate temperature $T_{S}^{0}$ corresponds to a constant deposition efficiency function $\epsilon_{0}\left(T_{S}^{0}\right)$ throughout the whole substrate surface.
- STEP 2. After an iteration $t=t_{0}+\Delta t$, the deposit profile is analytically predicted through Eq. 5. Nodes at the substrate surface, previously set at the locations $X_{0}(i), Y_{0}(i), Z_{0}(i)$ are thus transposed to a new set $X_{1}(i), Y_{1}(i), Z_{1}(i)$ (blue profile). Elements falling below the predicted analytical profile are activated within the deposit mesh.

- STEP 3. Both the newly activated deposit and substrate elements are subdivided into several subsets, based on their thermal boundary conditions. Elements are thus assigned to one of the differential Eqs. 7]13. It is during this step that, for the elements of deposit and substrate under direct contact with the impinging flame the convective heat transfer coefficient $h_{J}\left(r_{i}\right)$ and the surface temperature $T_{J}\left(r_{i}\right)$ are calculated based on the results of the flame characterisation stage.

- STEP 4. Within this step the nodal temperatures at the time step $t=t_{0}+\Delta t$ are determined by solving the system of differential heat diffusion equations at the previous time step $t_{0}$. The temperatures of the nodes at the target surface $\left(T_{S}^{i}\right)$ are then used to determine the deposition efficiencies $\epsilon\left(T_{S}^{i}\right)$ necessary to apply Eq. 5 for the subsequent time step.

- STEP 5. Equation 5 is again used to analytically predict the deposit shape in the next iteration $(t=$ $\left.t_{0}+2 \Delta t\right)$, based on the newly determined deposition 
STEP $1\left(t_{0}\right)$
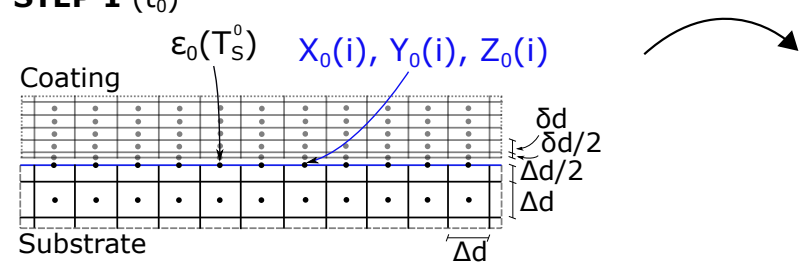

STEP $4\left(t_{0}+\Delta t\right)$

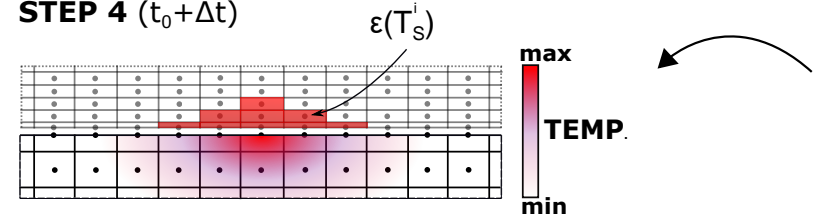

$\underset{\text { STEP } 5\left(\mathrm{t}_{0}+2 \Delta \mathrm{t}\right)}{\downarrow} \mathrm{X}_{2}(\mathrm{i}), \mathrm{Y}_{2}(\mathrm{i}), \mathrm{Z}_{2}(\mathrm{i})$

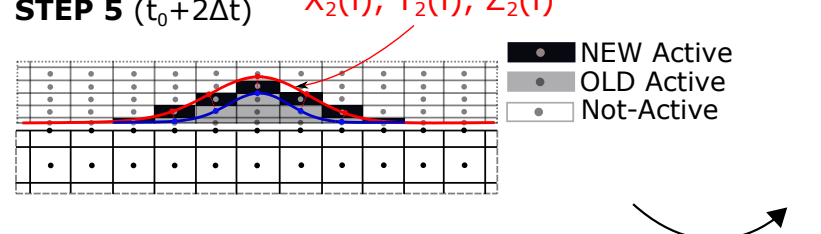

STEP $2\left(t_{0}+\Delta t\right)$
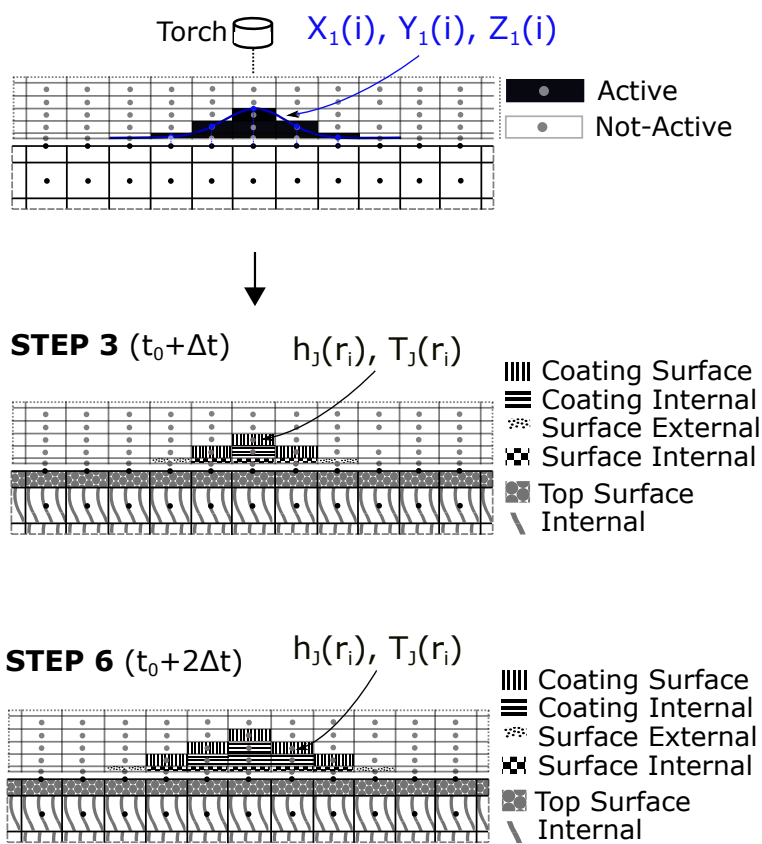

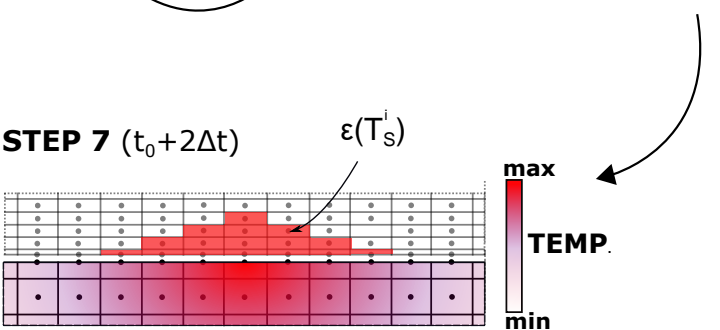

Figure 8: Schematic of the steps involved in the model numerical implementation during two subsequent time increments. In a repeated cycle, the deposit profile is analytically predicted based on the value of deposition efficiency $\left(\epsilon_{0}\left(T_{S}\right)\right)$ at the target surface (Steps 2 and 5 ). This latter parameter is determined from the surface temperature $T_{S}$, calculated by thermal analysis (Steps 4 and 7 ). In order to perform the thermal analysis, nodes falling below the analytical profiles are activated (Steps 2 and 5) and a heat diffusion equation among Eqs. 7.13 assigned to them (Steps 3 and 6).

efficiencies at the target surface $\epsilon\left(T_{S}^{i}\right)$. Previous deposit nodes at locations $\left(X_{1}(i), Y_{1}(i), Z_{1}(i)\right)$ (blue profile) are thus transposed to $\left(X_{2}(i), Y_{2}(i), Z_{2}(i)\right)$ (red profile), allowing new elements to be activated within the deposit mesh.

- STEP 6. The newly activated deposit elements, together with the ones activated in the previous step are re-arranged into new sets of heat diffusion equations 7, 13 based on their boundary conditions (e.g. elements previously belonging to the deposit surface will now become internal, etc.). The variation of deposit geometry also makes necessary to re-define the convective heat transfer coefficient $h_{J}\left(r_{i}\right)$ and surface temperature $T_{J}\left(r_{i}\right)$ for each of the nodes at the system surface, based on their profiles calculated within the flame characterisation stage.

- STEP 7. The new system of differential heat diffusion equations is solved to predict the overall system temperature at the new time step $t=t_{0}+2 \Delta t$. At this step, the deposition efficiency function $\epsilon\left(T_{S}^{i}\right)$ for the surface nodes can be re-calculated based on the new temperatures of the target surface nodes $\left(T_{S}^{i}\right)$. The procedure in the previous steps is then repeated in a loop until the total time set for the simulation is reached.

The relative simplicity of the fixed-, as opposed to complex adaptive-/stretched-meshes, generally employed for analogous simulations [31, guarantees easy programming and optimised computing times at no remarkable loss in shape prediction accuracy, even in regions of steep shape variation.

\section{Model validation and Discussions}

Hardware and experimental methodology employed, together with results and discussions from both model parameters evaluation and model implementation stages are presented in this section. 
The Castodyn ${ }^{\circledR}$ System 2000 torch controlled by a 6-axis robot $\left(\mathrm{ABB}^{\circledR}\right)$, Amperit 415.001 (HC-Starck ${ }^{\circledR}$ ) CoNiCrAlY powder and $50 \times 50 \times 6 \mathrm{~mm}$ stainless steel AISI 304 substrate material were employed in this work. The powder was selected for its widespread use as bondcoat layer for Thermal Barrier Coatings (TBC) in aerospace applications. For the sake of both computational and experimental simplicity, only the kinematic process parameter (i.e. torch feed speed $V$ ) has been varied in the range $10-100 \mathrm{~mm} / \mathrm{s}$ to validate the model. Conversely, operational process parameters: standoff distance, torchto-substrate inclination, gases pressure and flows, powder feed rate, powder and substrate materials and preparation have been maintained constant throughout the work. This is a reasonable approach since during the spraying process of a flat surface the energetic parameters (e.g. gases pressure, powder feed rates, etc.,) would be kept constant and only the torch feed speed would be changed to vary the amount of material deposited on the target surfaces. The values selected for these parameters are summarised in Tab. 11. It is worth noting that, for computational

Table 1: Process parameters used for model validation.

\begin{tabular}{|c|c|}
\hline Parameter (units) & Value \\
\hline \multicolumn{2}{|c|}{ Kinematic (varied) } \\
\hline Torch feed speed, V (mm/s) & $10-100$ \\
\hline \multicolumn{2}{|c|}{ Operational (fixed) } \\
\hline $\mathrm{C}_{2} \mathrm{H}_{2} / \mathrm{O}_{2}$ Flow rate $(\mathrm{slpm})$ & $15 / 34.1$ \\
\hline $\mathrm{C}_{2} \mathrm{H}_{2} / \mathrm{O}_{2}$ Pressure (Bar) & $0.8 / 3.8$ \\
\hline Compressed air pressure (Bar) & 3 \\
\hline Ar carrier gas flow (slpm) & 4 \\
\hline Powder type & CoNiCrAlY (Amperit 415.001) \\
\hline Powder feed rate, F (g/min) & 16 \\
\hline Standoff distance, SOD (mm) & 100 \\
\hline Torch/subst. inclination, $\Psi$ (deg) & 90 \\
\hline Numerical torch path length (mm) & 30 \\
\hline Substrate type & $50 \times 50 \times 6 \mathrm{~mm}$ AISI304 \\
\hline Substrate preparation & 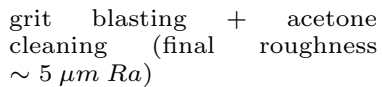 \\
\hline
\end{tabular}

expensiveness reasons, a $30 \mathrm{~mm}$ torch path over the substrate (in place of the whole $50 \mathrm{~mm}$ substrate length) was numerically modelled. Deposit shape have been measured by a 3 D light profilometer (Bruker ${ }^{\circledR}$ Contour Elite).

Physical properties of deposit and substrate, together with interface heat transfer coefficient and flame properties are reported in Tab. 2, It is worth noting that temperature-dependent properties are used for the substrate, while constant values are employed for the deposit due to the lack of literature data on these properties for thermally-sprayed CoNiCrAlY deposits. Additional parameters, not appearing in the analysis leading to Eqs. 7,13 for the sake of simplicity, are here also reported: droplets temperature at impact $\left(T_{D}^{\text {impact }}\right.$, measured by the authors using a Tecnar Accuraspray ${ }^{\circledR}$ G3C system), substrate initial temperature $\left(T_{Q}^{0}\right)$, surface fluid temperature and convective heat transfer coefficient at the substrate edges $\left(T_{E}\right.$ and $\left.h_{E}\right)$ and atmospheric temperature and convective heat transfer coefficient $\left(T_{A}\right.$ and $h_{A}$, this latter active at the un-sprayed substrate face).

Table 2: Deposit/substrate physical properties and boundary conditions used for thermal analysis.

\begin{tabular}{ll}
\hline Parameter (units) & Value \\
\hline Deposit (CoNiCrAlY) \\
$k_{D}\left(\frac{W K}{m}\right)[32$ & 4 \\
$\rho_{D}\left(\frac{k g}{m^{3}}\right)[32$ & 4500 \\
$C_{D}\left(\frac{J}{g K}\right)[33$ & 0.473 \\
$L_{D}\left(\frac{k J}{k g K}\right)[33$ & 293.7 \\
$\alpha_{D}(-)[34$ & 0.5 \\
$T_{D}^{i m p a c t}(K)$ & 2596 \\
Substrate $($ AISI & $304)$ \\
$k_{Q}\left(\frac{W}{m K}\right)[35$ & $6.22 \cdot 10^{-6} T^{2}+0.0031 T+13.07$ \\
$\rho_{Q}\left(\frac{k g}{m^{3}}\right)[35$ & $-5.436 \cdot 10^{-5} T^{2}-0.3516 T+8020$ \\
$C_{Q}\left(\frac{J}{g K}\right)[35$ & $-8.06 \cdot 10^{-3} T^{2}+1.845 \cdot 10^{-4} T+0.4164$ \\
$\alpha_{Q}(-)[36$ & 0.2 \\
$T_{Q}^{0}(K)$ & 300 \\
Boundary conditions & \\
$k_{i n t}\left(\frac{W}{m K}\right)$ & $k_{i n t}=2 k_{D} k_{Q} /\left(k_{D}+k_{Q}\right)$ \\
$h_{J}(r)\left(\frac{W}{m^{2} K}\right)$ & Fig. $10 \cdot \mathrm{a})$ \\
$T_{J}(r)(K)$ & Fig. $10 \cdot \mathrm{b})$ \\
$T_{E}(K)$ & 500 \\
$h_{E}\left(\frac{W}{m^{2} K}\right)$ & 1000 \\
$T_{A}(K)$ & 300 \\
$h_{A}\left(\frac{W}{m^{2} K}\right)$ & 100 \\
\hline &
\end{tabular}

In the following, results from the three stages of model parameters evaluation are presented, followed by the results of numerical model application.

\subsection{Model parameters evaluation}

\subsubsection{Validation of Flame characterisation approach $\left(h_{J}(r)\right.$ and $\left.T_{J}(r)\right)$}

An innovative setup was employed to measure the experimental substrate surface temperature $T_{S}^{E X P}(r, t)$ needed in this stage (Fig. 9). A $100 \times 100 \times 6 \mathrm{~mm}$ substrate was manufactured with 10 holes of $1 \mathrm{~mm}$ diameter drilled through its thickness and positioned at increasing radial positions from the centre ( 5 to $45 \mathrm{~mm}$, spaced $5 \mathrm{~mm}$ between each other). Type $\mathrm{K}$ sheathed grounded-junction thermocouples of $1 \mathrm{~mm}$ diameter were fitted within each hole, with their tip positioned in-plane with the top substrate surface (Fig. 9 inset). In order to improve the heat transfer to the thermocouple junction, a silver-based conductive paste was applied to the side walls of the thermocouples within the substrate channel. A tailored laser positioning system was designed to place the torch axis in the middle of the substrate and normally oriented in respect to the $\mathrm{x}-\mathrm{y}$ plane. By setting the torch properties as per Tab. 1. except for a static torch (i.e. feed speed $V=0 \mathrm{~mm} / \mathrm{s}$, due to the radial positioning of the thermocouples from the torch axis centre), the torch is then ignited and kept 


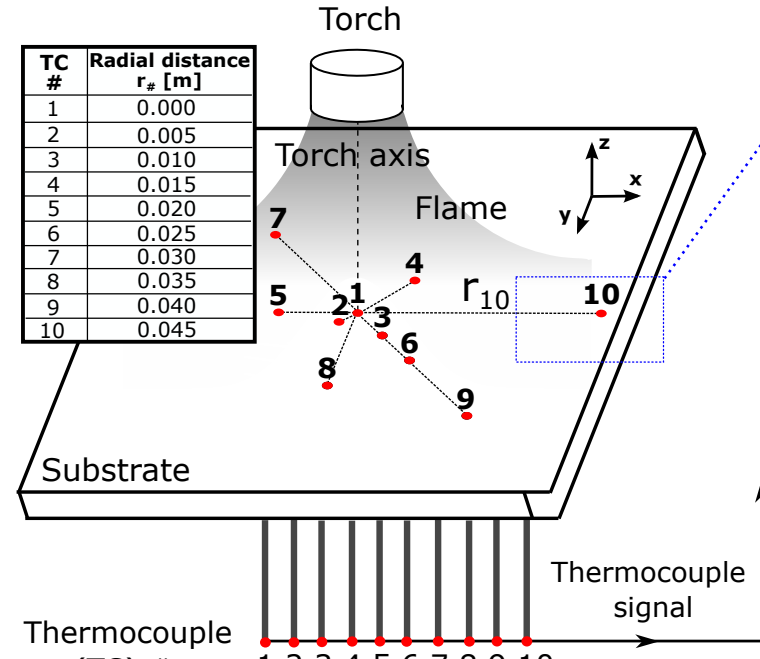

(TC) \#
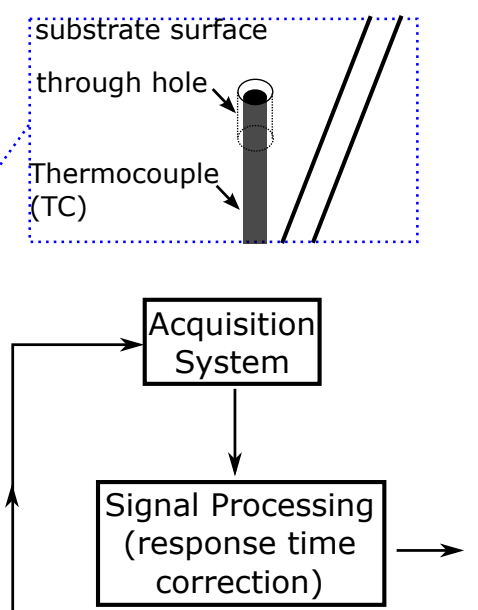

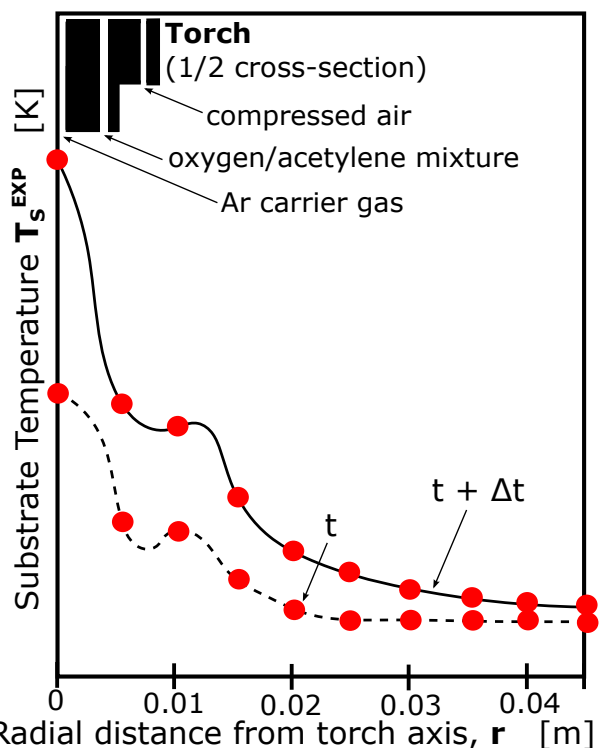

Figure 9: Schematic of the experimental apparatus used to measure the time-dependent substrate surface temperature $T T_{S}^{E X}(r, t)$ during the flame characterisation stage (Fig. 4). A static torch, whose axis is aligned with the substrate centre, is ignited and kept on for a set time span. A series of thermocouples are placed at equispaced radial positions (marked in red) from the torch axis.

on for 0.5 seconds, during which the thermocouples signal was recorded, at $1000 \mathrm{~Hz}$ sampling rate, by a 32-channels data-logger (Texas Instrument ${ }^{\mathrm{R}}$ SXI-1000). Due to the transient nature the thermocouple response, a signal processing technique was implemented to enhance the output signal based on the work of Baker et al. 37, assuming a first-order system:

$$
T_{t c}+\tau \frac{d T_{t c}}{d t}=T_{\text {real }}
$$

where $T_{t c}$ is the thermocouple signal, $T_{\text {real }}$ is the real temperature and $\tau=0.075 \mathrm{~s}$ is the response time (as specified by the manufacturer). The experimental surface temperature profiles $\left(T_{S}^{E X P}(r, t)\right)$ so obtained are then compared to analogous outputs from the simulation $\left(T_{S}^{S I M}(r, t)\right)$ until the convergence criteria set for the optimisation algorithm is achieved (see Fig. 4). The convective heat transfer coefficient $h_{J}^{F}(r)$ and temperature at surface $T_{J}^{F}(r)$ profiles, used as input in the simulations when the optimisation criteria is met, then represent the parameters required in this stage. These parameters, input as discrete set of points, are then spline-interpolated to provide continuous spatial functions.

The obtained flame surface parameters profiles, convective heat transfer coefficient $h_{J}^{F}(r)$ and temperature at surface $T_{J}^{F}(r)$, are depicted in Fig. 10 . The optimised convection heat transfer coefficient so determined (blue solid line, obtained as cubic polynomial interpolation), together with the initial profile used as input for the optimisation algorithm (from 22, dashed line), are depicted in Fig. 10(a). The obtained profile shows a scattered behaviour, possibly due to the turbulent flow experienced by the flame; however magnitudes are comparable to literature data on high Reynolds number jets impinging on flat surfaces 38.
It is also worth noting that the maximum value of heat transfer coefficient is located at approximately $10 \mathrm{~mm}$ radial distance, thus outside the stagnation zone. A specific explanation to this observation would require an in-depth analysis of the heat flux distribution for the experimental conditions of this study, which is not in scope for this specific work. In fact, it is commonly accepted in literature that the heat flux distribution for specific flame jet impingement problems is dependent on the complex interplay of a number of factors: nozzle-substrate spacing [39], Reynolds number, burner shape, equivalence ratio, oxygen enhancement [40, flame impingement angle 41] and interjets spacing (for multi-jet arrangements as in this study) [42, 43. The nozzle array configuration of this study, composed of a circular array of 15 burners, placed around an internal stream of powder-carrying Ar and surrounded by a circular array of ambient-temperature compressed air represents a complex interaction problem between jets of different temperatures, Reynolds number and chemical compositions, for which a simple analytical solution is non-trivial. However, the obtained spatially complex trend demonstrates the effectiveness of the adopted methodology in determining the flame boundary conditions, as opposed to complex analytical solutions which would have to be derived and validated each time a new torch design and/or deposition parameters are employed. Similarly, magnitude of the optimised temperature at surface (blue solid line in Fig. 10(b), obtained as spline interpolation) is comparable to similar gradients measured in literature on oxyacetylene spray systems 44. It is worth noting that a local minimum is observed in the profile at $\sim 5 \mathrm{~mm}$ radial distance, while a local maximum is present at $\sim 10 \mathrm{~mm}$. While the former is likely generated by the cold sheath of compressed air surrounding the array of burner flames, the interaction 


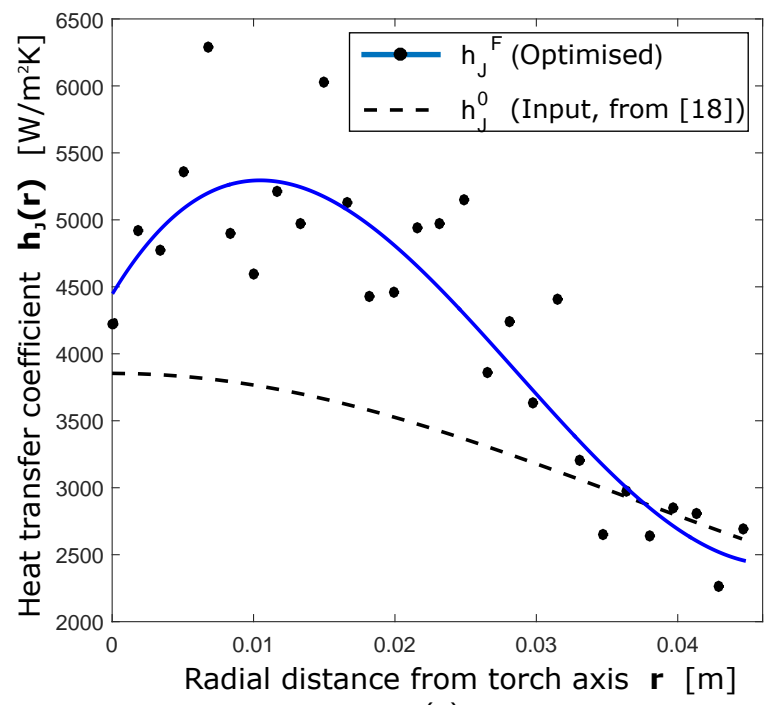

(a)

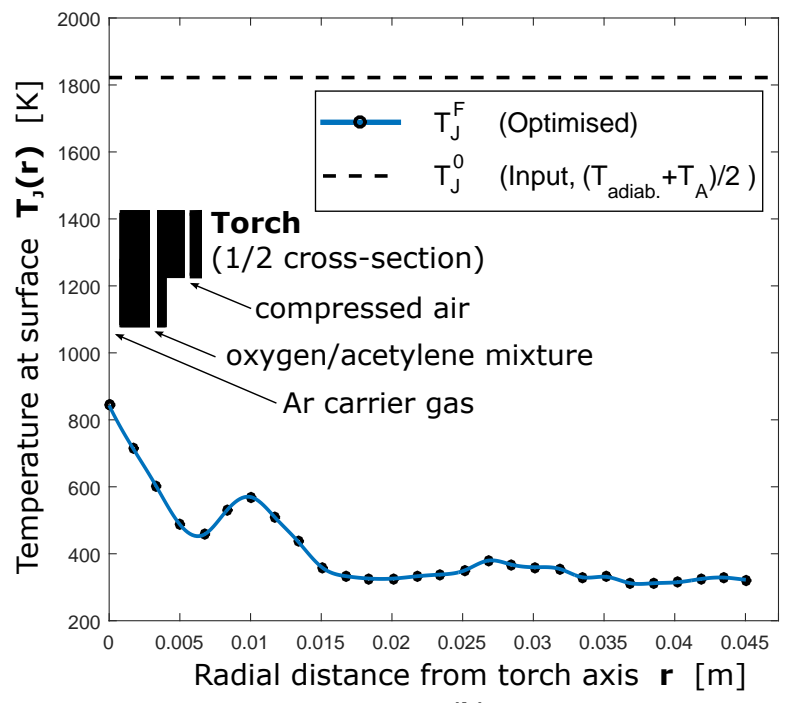

(b)

Figure 10: (a) Flame convective heat transfer coefficient $h_{J}(r)$ and (b) temperature at surface $\left(T_{J}(r)\right.$ profiles, obtained from an optimisation algorithm within the flame characterisation stage. Optimised (solid line) and input (dashed line) profiles are depicted for both parameters, with cubic polynomial and spline-interpolation performed in (a) and (b) respectively to obtain the optimised profiles. The convective heat transfer coefficient is observed to closely follow the initial theoretical prediction only at higher values of radial distance. The difference between input and optimised values of surface temperature demonstrates the prediction quality of the optimization algorithm even at input values far from the actual solution. A schematic 2D cross-sectional view of the torch in respect to the temperature profile, shows that the sheath of compressed air is likely responsible for the value decrease observed at $r \sim 5 \mathrm{~mm}$.

between the complex jets of this work, as previously explained, is likely to generate local instabilities, giving rise to the observed local maximum. However, in the same figure, the difference between the input temperature value (calculated as $\left.T_{J}^{0}=\left(T_{\text {adiab. }}+T_{A}\right) / 2=1850 \mathrm{~K}\right)$ and the optimised experimental profile demonstrates the validity of the proposed approach in predicting solutions even if the initial input solution is far from the real one.

\subsubsection{Validation of Calibration approach (plume distribu- tion parameters $A$ and $b$ )}

The application of Eq. 16 to a deposit cross-section $Z(r)$ sprayed at a torch feed speed $V=100 \mathrm{~mm} / \mathrm{s}$ and other deposition parameters as in Tab. 1. allowed the deposition rate $\dot{D}(r)$ depicted in Fig. 11 (black line) to be determined. The curve fit, based on Eq. 2 is also overlapped on the same graph (red dashed line). From this interpolation, and a (weighted) measured deposition efficiency $\epsilon=82.8 \%$, values $A=0.0018 \mathrm{~m} / \mathrm{s}$ and $b=9.16 \cdot 10^{4} \mathrm{~m}^{-2}$ were obtained for the plume distribution parameters. Since any torch feed speed could in principle be used for calibration purposes (provided that a sufficiently high value is used in order to make use of Eq. 16 and that the respective deposition efficiency is weight-measured accordingly), plume distribution parameters were also evaluated from other nine deposits sprayed at feed speeds from 20 to 100 $\mathrm{mm} / \mathrm{s}$. The sample mean resulted in $A=0.0017 \mathrm{~m} / \mathrm{s}$ and $b=8.21 \cdot 10^{4} \mathrm{~m}^{-2}$ with standard deviations of $0.00008 \mathrm{~m} / \mathrm{s}$ and $5.26 \cdot 10^{3} \mathrm{~m}^{-2}$ respectively, demonstrating the low variability in the proposed calibration approach.

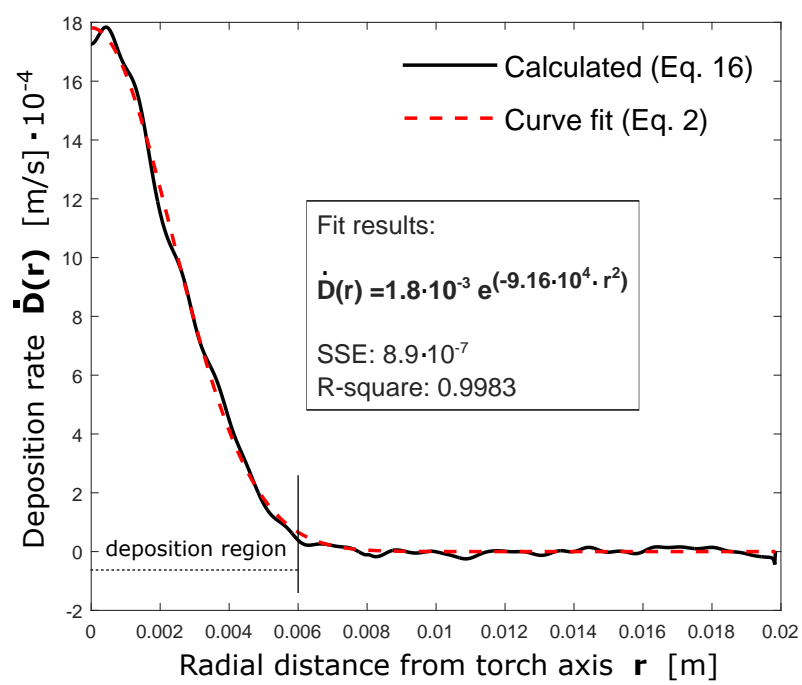

Figure 11: Deposition rate profile (black) and Gaussian interpolation (red) obtained in the calibration stage. The width of the deposition region (i.e. the radius from the torch axis outside which no material is deposited, used for surface temperature averaging in Fig. 57, is also specified by a black dashed line.

\subsubsection{Validation of Deposition Efficiency evaluation ap- proach $\left(\epsilon\left(T_{S}\right)\right)$}

Two deposits have been sprayed at the lower $\left(V_{1}=\right.$ $10 \mathrm{~mm} / \mathrm{s})$ and higher $\left(V_{f}=100 \mathrm{~mm} / \mathrm{s}\right)$ range of torch feed speed employed in this study, by maintaining the operational process parameters as per Tab. 11. For the two specimens, deposition efficiencies of $\epsilon_{1}=86.5$ and $\epsilon_{f}=82.8 \%$, are determined respectively by weighting measurement. The corresponding averaged surface temperatures (refer 


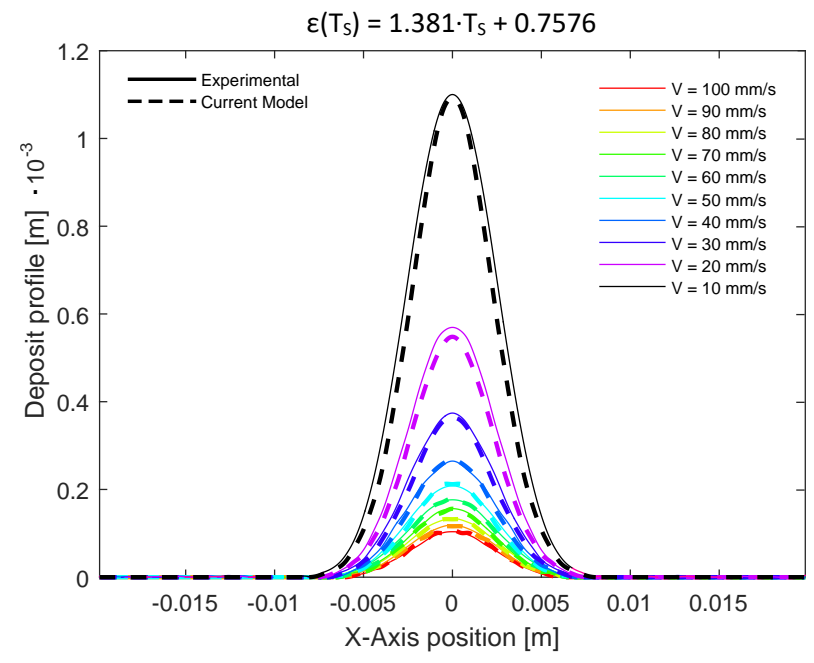

(a)

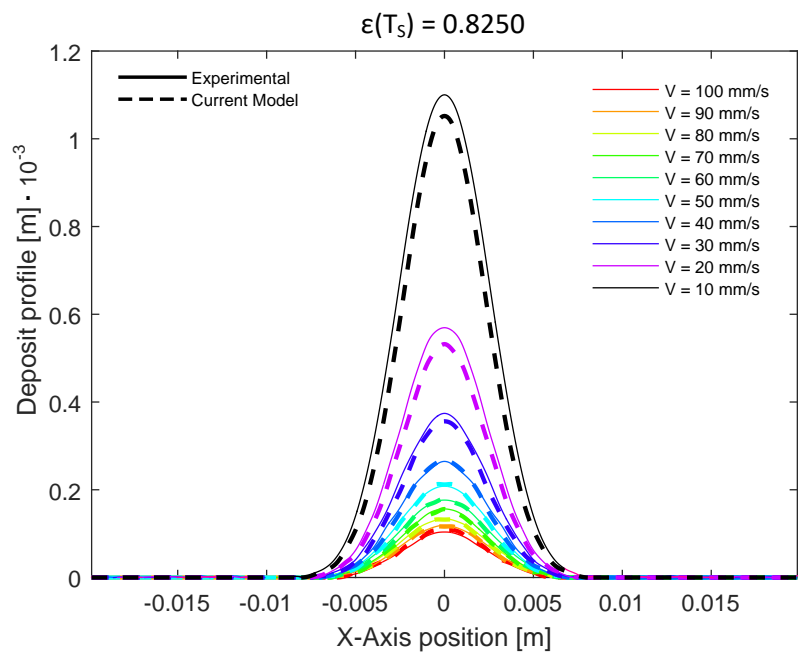

(b)

Figure 12: Comparison between experimental (solid line) and numerical (dashed line) 2D cross-sectional profiles of deposits sprayed at different torch feed speeds V. A better match is obtained by using (a) the deposition efficiency expression determined in this work as opposed to (b) a constant value obtained by weighted measurement.

to the discussion following Fig. 5 for details), as determined by numerical simulations, gave $T_{S 1}=778$ and $T_{S f}=510 K$ respectively. If a linear interpolation of the form $\epsilon=a T_{S}+b$ is performed from the two points just determined, a continuous function of the form:

$$
\epsilon\left(T_{S}\right)=1.381 \cdot 10^{-4} \cdot T_{S}+0.7576
$$

is thus obtained. Although it could be argued that a linear interpolation represent an oversimplification of deposition efficiency behaviour in respect to the real profile, it is demonstrated here that its application brings accurate results. Moreover, due to the lack of literature data in this area, no direct comparison could be undertaken. However, analogy to spray forming process shows that experimentally-determined deposition efficiency, although elevated data scattering is commonly experienced, always exhibits (as within this work) an increasing trend with surface temperature 45 .

\subsection{Model application}

In order to investigate the validity of the proposed model, ten deposits were experimentally sprayed and numerically simulated at torch feed speed intervals of 10 $\mathrm{mm} / \mathrm{s}$ within the $10-100 \mathrm{~mm} / \mathrm{s}$ range adopted in the study, with other deposition parameters as in Tab. 1. Fig. 12(a) shows the comparison between experimental (solid line) and numerical (dotted line) cross-sectional profiles, calculated as mean height from several cross-sections over the whole deposit length and obtained assuming the temperature-dependent deposition efficiency profile in Eq. 18. The simulated curves closely match the experimental profiles, with a maximum difference in terms of total cross-sectional area of $<5 \%$ and decreasing at higher feed speeds, where surface temperature, and thus deposition efficiency, is expected to show small variability. This latter effect can be appreciated by plotting the numerically determined average surface temperatures (and corresponding deposition efficiencies calculated by using the linear interpolation of Eq. 18) versus torch feed speed (Fig. 13). It is clear from the graph that the deposition efficiency maintains a roughly constant value at high feed speeds $(\sim 70-100 \mathrm{~mm} / \mathrm{s})$, while shows a steep change at lower values. The critical role of deposition efficiency is appreci-

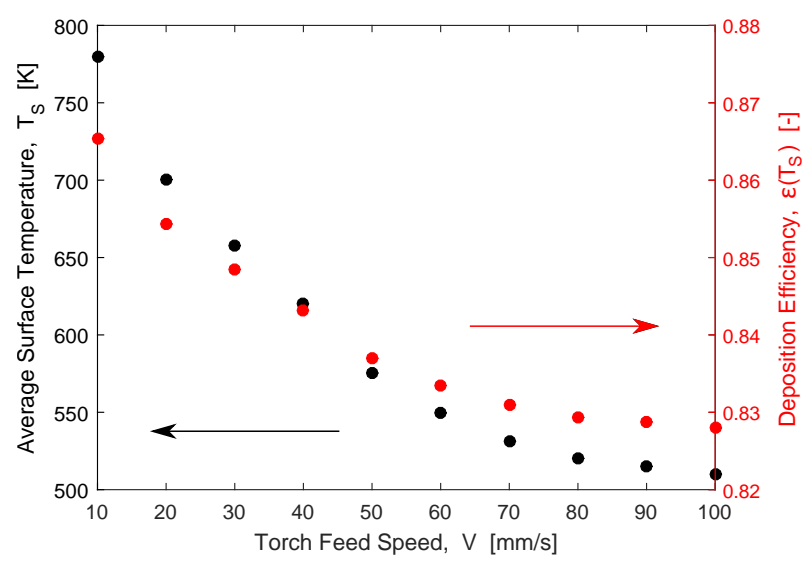

Figure 13: The deposition efficiency shows low fluctuation at high torch feed speeds. This is linked to the low variation in surface temperature at the point of droplets impact (black, left) at these torch feed speeds, as obtained from averaging of numerical simulations within the deposition area around the torch axis (see Fig. 5).

ated by analysing the results of numerical simulations performed assuming a constant (as opposed to temperaturedependent) value of this parameter, corresponding to the weighted measurement result of the deposit sprayed at $V=100 \mathrm{~mm} / \mathrm{s}$, i.e. $\epsilon=82.50 \%$ (Fig. $12(\mathrm{~b})$ ). A difference is clearly observed between the simulated and experimental profiles, reaching $\sim 15 \%$ in cross-sectional area at the lower feed speeds $(V=10-20 / ; \mathrm{mm} / \mathrm{s})$ and de- 


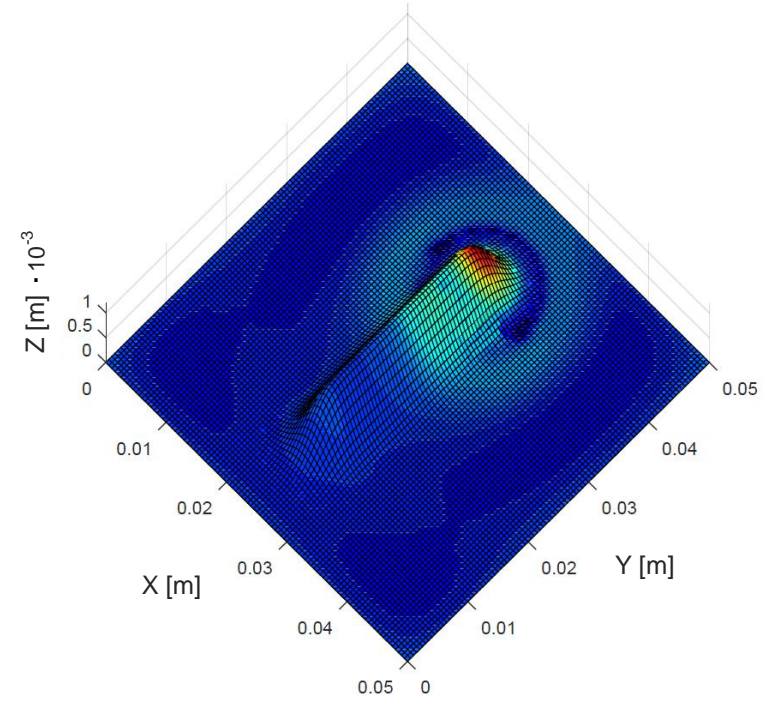

(a)

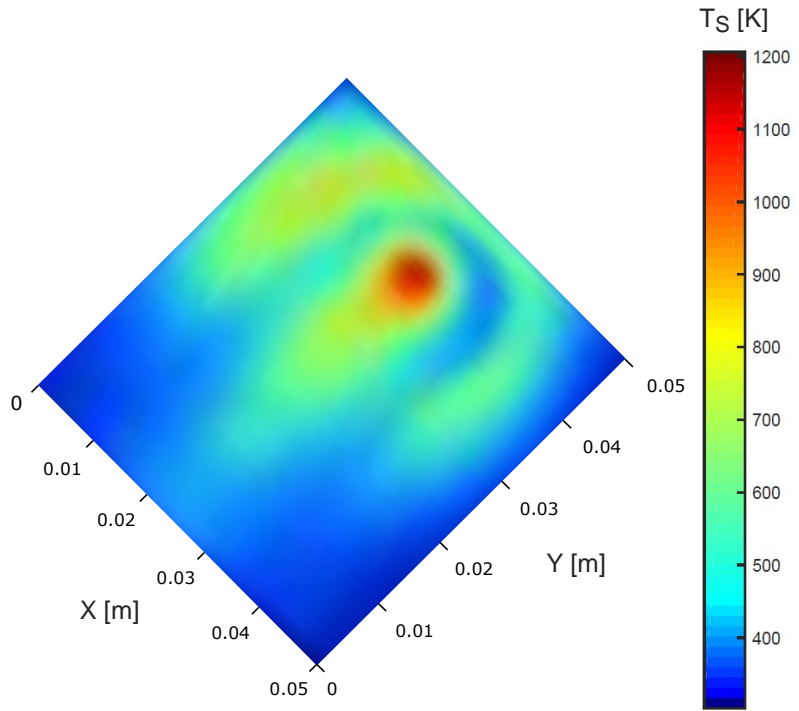

(b)

Figure 14: (a) Simulated and (b) experimental distributions of surface temperature $T_{S}$. A similar spatial distribution is observed in both cases, with higher magnitudes measured in the experimental case due to signal screening from the flame during IR measurement. This also explains the higher scatter in temperature distribution within the cold zone around the hot spot in the simulated case as compared to the homogeneous distribution of the experimental result.

creasing in magnitude for deposits sprayed at higher feed speed.

It is worth mentioning that the deposition impact angle $(\theta$, inset of Fig. 2) calculated for the produced deposits shows a maximum of $\sim 15^{\circ}$ for the thickest deposit $(V=$ $10 \mathrm{~mm} / \mathrm{s}$ ). According to the work of J. Mi et al., on spray forming [17, the geometric contribution to the deposition efficiency is negligible for impact angles $<30^{\circ}$. This would justify the assumption made from Eq. 5 to 6 of negligible influence of the impact angle on the mechanical interaction term $\epsilon(\eta)$.

In order to test the validity of the proposed model in predicting the system temperature, the surface temperature of numerical and experimental deposits, sprayed at $V=10 \mathrm{~mm} / \mathrm{s}$, are depicted in Figs. 14(a) and 14(b) respectively. The experimental surface temperatures were measured by placing an IR thermal camera statically in front of the substrate during deposition. A $9 \mathrm{~Hz}$ refresh rate Fluke ${ }^{\circledR}$ Ti450 thermal imaging camera, calibrated in the -20 to $+1200^{\circ} \mathrm{C}$ range was employed for the scope, assuming a constant material emissivity $\epsilon=0.5$ (as employed in the model for CoNiCrAlY, Tab. 2 and $100 \%$ atmosphere transmittance. A peak temperature of $\sim 1200 \mathrm{~K}$ is measured in the hot region of both systems. This could have been also extracted by the peak temperature measured in Fig. 5, showing the surface temperature profiles at subsequent time steps during the simulation for the same experiment. Around the hot spot, a circular cold zone is noted, corresponding to the shoulder observed in the flame surface temperature profile $\left(T_{J}(r)\right)$ of Fig. 10 and likely caused by a combination of the compressed air sheath surrounding the torch burners. The magnitude and extension of this cold zone however, differ between the two results, with higher temperature measured by means of IR imaging as compared to numerical analysis. Moreover, the temperature at the deposit surface show a general higher magnitude ( $\sim 0-300^{\circ} \mathrm{C}$ depending on the location) over the whole IR-profile as compared to the simulated one. It should be noted that care has to be taken when quantitatively interpreting IR-measured data 46]. The total radiation received by the IR sensor is in fact a combination between the direct emission of the target object (result of a temperature-, surface condition-, emitting angle- and alloy composition-dependent emissivity) and contributions linked to reflected radiation and atmospheric emission. In the process here analysed, the flame is interposed between the deposit and the thermal sensor, thus partially absorbing and concurrently emitting unwanted radiative components. This explains why the scattered temperature distribution measured within the cold ring around the hot spot in the simulated case (and partially extending also on the deposit bump in, Fig. 14(a)) is not measured in the experimental case of Fig. 14(b). An ongoing in-depth study is being performed in order to address the aforementioned effects.

As a final analysis, the microstructure of deposits sprayed at the two extremes of the torch feed speed range employed (e.g. $V=10$ and $100 \mathrm{~mm} / \mathrm{s}$ ) was assessed. Optical microscopies of the two cross-sections are depicted in Figs. 15(a) $(V=10 \mathrm{~mm} / \mathrm{s})$ and 15(b) $(V=100 \mathrm{~mm} / \mathrm{s})$. The two deposits show a comparable microstructure in terms of porosity, unmolten particles and oxide strings, as expected from CFS of CoNiCrAlY materials [19]. This similarity in microstructure between the two specimens thus confirms the validity of assumption made on constant deposit density $\rho_{D}$ employed throughout the model $\left(\rho_{D}\right.$ 

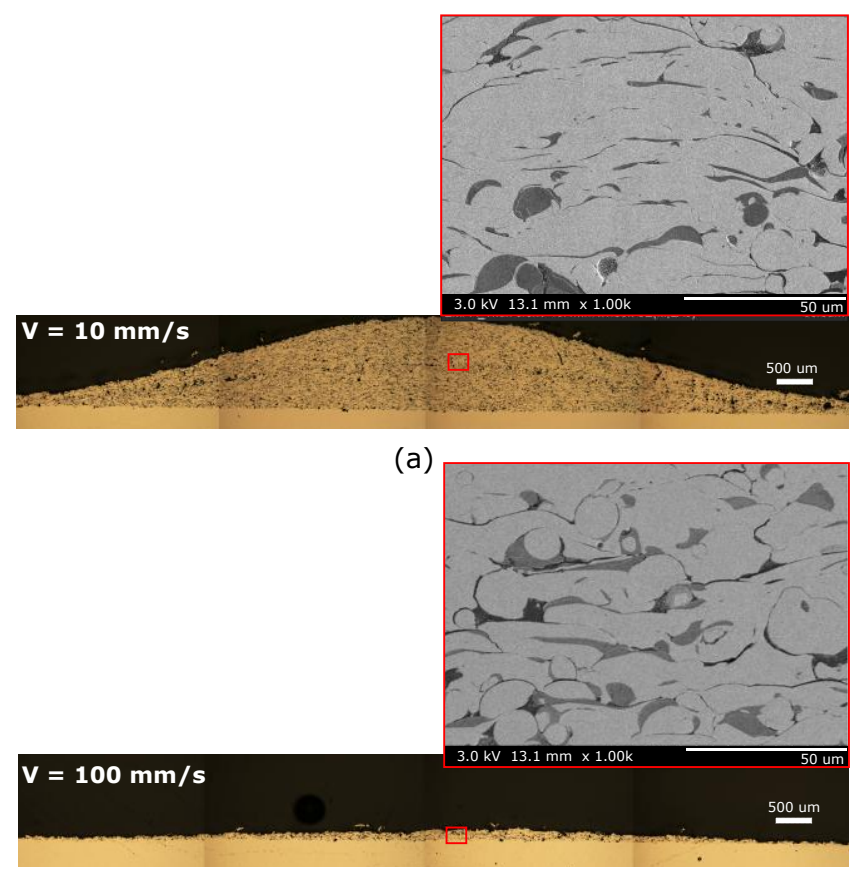

(b)

Figure 15: Optical micrographs of deposit 2D cross-sections, obtained at (a) $\mathrm{V}=10 \mathrm{~mm} / \mathrm{s}$ and (b) $\mathrm{V}=100 \mathrm{~mm} / \mathrm{s}$. In inset, SEM micrographs of the two coatings show similar microstructure in terms of porosity, unmolten particles and oxide strings, suggesting a similar deposit density $\rho_{D}$, as assumed throughout the model.

has been embedded in the plume distribution parameter $A$ in Eq. 6].

\section{Summary and Conclusions}

A novel modelling approach for the prediction of timedependent, three-dimensional shape of deposits produced via thermal spray methods is proposed. In contrast with conventional models, based on the stochastic thermalphysical properties of the impacting droplet plume, the approach here developed is flexible in which the continuous deposition efficiency function and other spray distribution parameters, necessary for deposit shape prediction, are determined and stored in a preliminary calibration phase to be re-used for predictions at any arbitrary value of torch feed speed. This calibration step is essential to capture complex deposition parameters, rapidly varying in space and with deposition hardware and materials, thus not easily described in pure analytical terms.

The model framework, validated on a generic energy beam process for coating deposition (i.e. combustion flame spray), distinguishes itself through the following key findings:

- It allows the time-dependent prediction of deposit shape in three-dimensions with a high dimensional accuracy $(\sim 10-100 \mu \mathrm{m})$. This opens the possibility to simulate coatings of variable thickness by control over the torch feed speed, i.e. a parameter that can be easily controlled with modern manipulators/robots.

- Accurate deposit predictions are enabled by the characterisation of the deposition efficiency function, considered dependent primarily on the temperature of the target surface. This is demonstrated by a $10 \% \mathrm{im}-$ provement difference between simulated and experimental deposits cross-sectional area, measured using a temperature-dependent deposition efficiency function as opposed to a constant value. Moreover, this difference in cross-sectional area shows a marked decrease (from $\sim 15 \%$ to $<5 \%$ ) when deposits are sprayed at high feed speeds. This is due to the lower deposition efficiency variability caused by a corresponding similar surface temperature measured at these elevated feed speeds. The deposition efficiency function is determined in a preliminary stage of model application through a newly-developed semi-empirical method.

- A novel optimisation-based algorithm, utilising as minimisation function the difference between simulated and experimental substrate surface temperatures, is employed to determine the flame properties (convective heat transfer coefficient and temperature at surface). This has been proven to provide a useful tool to determine, with accurate spatial accuracy, these parameters which often remain unknown in forced convection heat transfer problems.

- The deposition rate for the process can be accurately determined by a simple calibration approach, avoiding the need for complex modelling based on the stochastic nature of thermal, velocity and spatial distribution of the droplets plume.

- The numerical implementation of the model is performed via an implicit finite-differences method, providing a stable and computationally cheap prediction tool. However, care must be taken when comparing numerically-obtained surface temperatures with experimental IR measurements due to the screening effect given by the flame in this latter case. Differences in the range 0 to $300^{\circ} \mathrm{C}$ have been measured between results of the two approaches.

The model has thus been proven capable of accurately predicting, in the time domain, the three-dimensional shape of deposits through the inclusion of droplets-substrate interaction effects in the form of a deposition efficiency function. This makes the model ideal for the prediction of deposit growth onto patches of delaminated coating present in difficult-to-spray components, where complex deposition hardware motion patterns are required (i.e. repair operations). Moreover, the flexibility given by the newlydeveloped calibration approach for the determination of fundamental model parameters, allows the model to be applied to several thermal spray technologies and deposition parameters, thus providing an efficient tool in many 
engineering fields as aerospace, power generation, and automotive industries.

\section{Acknowledgments}

The authors would like to thank Drs. J. Kell and A.D. Norton for their support throughout the work development and Mr. R. Screaton of the University of Nottingham for support on setting up the deposition hardware. The work has been supported by EPSRC UK (EP/L505572/1) under a Rolls-Royce CASE award scheme.

\section{References}

[1] H. Herman, S. Sampath, R. McCune, Thermal spray: current status and future trends, MRS bulletin 25 (2000) 17-25.

[2] C.-J. Li, W.-Y. Li, Y.-Y. Wang, G.-J. Yang, H. Fukanuma, A theoretical model for prediction of deposition efficiency in cold spraying, Thin Solid Films 489 (2005) 79-85.

[3] H. Chen, S. W. Lee, H. Du, C. X. Ding, C. H. Choi, Influence of feedstock and spraying parameters on the depositing efficiency and microhardness of plasma-sprayed zirconia coatings, Materials Letters 58 (2004) 1241-1245.

[4] D. L. Gilmore, R. C. Dykhuizen, R. A. Neiser, T. J. Roemer, M. F. Smith, Particle velocity and deposition efficiency in the cold spray process, Journal of Thermal Spray Technology 8 (1999) 576-582.

[5] M. Pasandideh-fard, R. Bhola, S. Chandra, J. Mostaghimi, Deposition of tin droplets on a steel plate: simulations and experiments, Heat and Mass Transfer 41 (1998) 2929-2945.

[6] M. Xue, Y. Heichal, S. Chandra, J. Mostaghimi, Modeling the impact of a molten metal droplet on a solid surface using variable interfacial thermal contact resistance, Journal of Materials Science 42 (2006) 9-18.

[7] C. Kang, H. Ng, Splat morphology and spreading behavior due to oblique impact of droplets onto substrates in plasma spray coating process,, Surface and Coatings Technology 200 (2006) 5462-5477.

[8] K. Remesh, H. W. Ng, S. C. M. Yu, Influence of process parameters on the deposition footprint in plasma-spray coatings, Journal of Thermal Spray Technology 12 (2003) 377-392.

[9] R. Ghafouri-Azar, J. Mostaghimi, S. Chandra, M. Charmchi, A stochastic model to simulate the formation of a thermal spray coating, Journal of Thermal Spray Technology 12 (2003) 53-69.

[10] M. Xue, S. Chandra, J. Mostaghimi, C. Moreau, A stochastic coating model to predict the microstructure of plasma sprayed zirconia coatings, Modelling and Simulation in Materials Science and Engineering 16 (2008) 1-19.

[11] S. Chandra, P. Fauchais, Formation of solid splats during thermal spray deposition, Journal of Thermal Spray Technology 18 (2009) 148-180.

[12] J. Xie, D. Nelias, H. W.-L. B. andK. Ogawa, Y. Ichikawa, Simulation of the cold spray particle deposition process, Journal of Tribology 137(4).

[13] W. Y. Li, K. Yang, S. Yin, X. P. Guo, Numerical analysis of cold spray particles impacting behavior by the eulerian method: A review, Journal of Thermal Spray Technology 25 (2016) 14411460.

[14] A. Manap, T. Okabe, K. Ogawa, Computer simulation of cold sprayed deposition using smoothed particle hydrodynamics, Procedia Engineering 10 (2011) 1145-1150.

[15] M. Yu, W. Y. Li, F. F. Wang, H. L. Liao, Finite element simulation of impacting behavior of particles in cold spraying by Eulerian approach, Journal of Thermal Spray Technology 21 (2012) 745-752.

[16] P. Mathur, S. Annavarapu, D. Apelian, A. Lawley, Spray casting: an integral model for process understanding and control, Materials Science and Engineering A 142 (1991) 261-276.
[17] J. Mi, P. Grant, Modelling the shape and thermal dynamics of Ni superalloy rings during spray forming. Part 1: Shape modelling - droplet deposition, splashing and redeposition., Acta Materialia 56 (2008) 1588-1596.

[18] C. Cui, U. Fritsching, A. Schulz, Q. Li, Mathematical modeling of spray forming process of yubular preforms Part 1. Shape evolution, Acta Materialia 53 (2005) 2765-2774.

[19] F. Fanicchia, D. Axinte, J. Kell, R. McIntyre, G. Brewster, A. D. Norton, Combustion flame spray of CoNiCrAlY \& YSZ coatings, Surface and Coatings Technology , 'in press'.

[20] P. L. Fauchais, J. V. R. Heberlein, M. I. Boulos, Thermal spray fundamentals. From powder to part, Springer, 2014.

[21] F. Incropera, D. P. Dewitt, T. L. Bergman, A. S. Lavine, Introduction to Heat Transfer, John Wiley \& Sons, 2007.

[22] H. Martin, Heat and mass transfer between impinging gas jets and solid surfaces, Advances in Heat Transfer 13 (1977) 1-60.

[23] M. J. Remie, M. F. G. Cremers, K. R. A. M. Schreel, L. P. H. de Goey, Analysis of the heat transfer of an impinging laminar flame jet, International Journal of Heat and Mass Transfer 50 (2007) 2816-2827.

[24] M. Remie, G. Särner, M. F. G. Cremers, A. Omrane, K. R. A. M. Schreel, M. Alden, L. P. H. de Goey, Extended heattransfer relation for an impinging laminar flame jet to a flat plate, international Journal of Heat and Mass Transfer 51 (2008) 1854-1865.

[25] G. K. Agrawal, S. Chakraborty, S. K. Som, Heat transfer characteristics of premixed flame impinging upwards to plane surfaces inclined with the flame axis jet, International Journal of Heat and Mass Transfer 53 (2010) 1899-1907.

[26] J. Appel, H. Bockorn, M. Frenklach, Kinetic modeling of soot formation with detailed chemistry and physics: laminar premixed flame of $c_{2}$ hydrocarbons, Combustion and Flame 121 (2000) 122-136.

[27] J. C. Lagarias, J. A. Reeds, M. H. Wright, P. E. Wright, Convergence properties of the nelder-mead simplex method in low dimensions, SIAM Journal of Optimisation 9 (1998) 112-147.

[28] A. Ozturk, B. M. Cetegen, Modeling of precipitate formation in precursor droplets injected axially into an oxygen/acetylene combustion flame, Materials Science and Engineering A 422 (2006) 163-175.

[29] A. D. Hewitt, Technology of oxy-fuel gas prprocess. Part 2: Comparative combustion properties of fuel gases, Welding 40 (1972) 382-389.

[30] D. A. Axinte, D. S. Srinivasu, J. Billingham, M. Cooper, Geometrical modemodel of abrasive waterjet footprints: A study for $90^{\circ}$ jet impact angle, CIRP Annals - Manufacturing Technology 59 (2010) 341-346.

[31] J. Mi, P. S. Grant, Modelling the shape and thermal dynamics of Ni superalloy rings during spray forming. Part 2: Thermal modelling - heat flow and solidification., Acta Materialia 56 (2008) 1597-1608.

[32] R. R. Holmes, T. N. McKechnie, Vacuum application of thermal barrier plasma coatings. In NASA Marshall Space Flight Center Advanced Earth-to-Orbit Propulsion Technology vol 1, Richmond, 1988.

[33] Y. M. Yang, H. Liao, C. Coddet, Simulation and Application of a HVOF Process for MCrAlY Thermal Spraying, Journal of Thermal Spray Technology 11 (2002) 36-43.

[34] V. Texeira, M. Andritschky, W. Fischer, H. P. Buchkremer, D. Stöver, Effects of deposition temperature and thermal cycling on residual stress state in zirconia-based thermal barrier coatings, Surface and Coatings Technology 120-121 (1999) 103111.

[35] D. Deng, H. Murakawa, Numerical simulation of temperature field and residual stress in multi-pass welds in stainless steel pipe and comparison with experimental measurements, Computational Materials Science 37 (2006) 269-277.

[36] L. Gardner, K. T. Ng, Temperature development in structural stainless steel sections exposed to fire, Fire Safety Journal 41 (2006) 185-203.

[37] H. D. Baker, E. A. Ryder, N. H. Baker, Temperature measure- 
ment in engineering. Vol. 2, Stamford: Omega Press, 1961.

[38] V. Katti, S. V. Prabhu, Experimental study and theoretical analysis of local heat transfer distribution between smooth flat surface and impinging air jet from a circular straight pipe nozzle, International Journal of Heat and Mass Transfer 51 (2008) $4480-4495$.

[39] V. Hindasageri, R. P. Vedula, S. V. Prabhu, Heat transfer distribution for three interacting methaneair premixed impinging flame jets, International Journal of Heat and Mass Transfer 88 (2015) 914-925.

[40] C. E. Baukal, B. Gebhart, Heat transfer from axygen enhanced/natural gas flame impinging a normal plane surface, Experimental Thermal and Fluid Science 16 (1998) 247-259.

[41] S. S. Hou, Y. C. Ko, Influence of oblique angle and heating height on flame structure, temperature field and efficiency of an impinging laminar jet flame, Energy Conversion and Management 46 (2005) 941-958.

[42] L. L. Dong, C. W. Leung, C. S. Cheung, Heat transfer characteristics of a pair of impinging rectangular flame jets, Journal of Heat Transfer 125 (2003) 1140-1146.

[43] S. Chander, A. Ray, Heat transfer characteristics of three interacting methane/air flame jets impinging on a flat surface, International Journal of Heat and Mass Transfer 50 (2007) 640-653.

[44] A. Ozturk, B. M. Cetegen, Morphology of ceramic particulates formed in a premixed oxygen/acetylene flame from liquid precursor droplets, Acta Materialia 53 (2005) 2531-2544.

[45] M. Buchholz, V. Uhlenwinkel, N. Ellendt, The effect of deposit temperature on the sticking efficiency during spray forming, in: Proc: 4th Int. Conf. On Spray Forming, 1999.

[46] D. C. Hofmann, S. N. Roberts, H. Kozachkov, Infrared thermal processing history of a Ti-based bulk metallic glass matrix composite manufactured via semi-solid forging, Acta Materialia 95 (2015) 192-200 